\title{
Tackling light higgsinos at the ILC
}

\author{
Mikael Berggren ${ }^{1}$, Felix Brümmer ${ }^{1}$, Jenny List ${ }^{1}$, Gudrid Moortgat-Pick ${ }^{2}$, Tania Robens ${ }^{3}$, Krzysztof Rolbiecki ${ }^{4,5}$, \\ Hale Sert ${ }^{1,2}$ \\ ${ }^{1}$ DESY, Notkestraße 85, 22607 Hamburg, Germany \\ ${ }^{2}$ University of Hamburg, Physics Department, Luruper Chaussee 149, 22761 Hamburg, Germany \\ ${ }^{3}$ IKTP, TU Dresden, Zellescher Weg 19, 01069 Dresden, Germany \\ ${ }^{4}$ IFT-UAM/CSIC, C/ Nicolás Cabrera 13-15, 28049 Madrid, Spain \\ ${ }^{5}$ IFT, University of Warsaw, ul. Hoża 69, 00681 Warsaw, Poland
}

DESY 13-098

\begin{abstract}
In supersymmetric extensions of the Standard Model, higgsino-like charginos and neutralinos are preferred to have masses of the order of the electroweak scale by naturalness arguments. Such light $\widetilde{\chi}_{1}^{0}, \widetilde{\chi}_{2}^{0}$ and $\widetilde{\chi}_{1}^{ \pm}$states can be almost mass degenerate, and their decays are then difficult to observe at colliders. In addition to the generic naturalness argument, light higgsinos are well motivated from a top-down perspective. For instance, they arise naturally in certain models of hybrid gauge-gravity mediation. In the present analysis, we study two benchmark points which have been derived in the framework of such a model, which exhibit mass differences of $\mathcal{O}(\mathrm{GeV})$ in the higgsino sector. For chargino pair and neutralino associated production with initial-state photon radiation, we simulate the detector response and determine how accurately the small mass differences, the absolute masses and the cross sections can be measured at the International Linear Collider. Assuming that $500 \mathrm{fb}^{-1}$ has been collected at each of two beam-polarisations $P\left(e^{+}, e^{-}\right)=( \pm 30 \%, \mp 80 \%)$, we find that the mass differences can be measured to $40-300 \mathrm{MeV}$, the cross sections to $2-5 \%$, and the absolute masses to $1.5-3.3 \mathrm{GeV}$, where the range of values correspond to the different scenarios and channels. Based on these observables we perform a parameter fit in the MSSM, from which we infer that the higgsino mass parameter $\mu$ can be measured to a precision of about $\Delta \mu=2-7 \mathrm{GeV}$. For the electroweak gaugino mass parameters $M_{1}, M_{2}$, which are chosen in the multi- $\mathrm{TeV}$ range, a narrow region is compatible with the measurements. For both parameters independently, we can determine a lower bound.
\end{abstract}

\section{Introduction}

The LHC experiments have discovered a Standard Model (SM)-like Higgs boson with $m_{h} \approx 126 \mathrm{GeV}[1,2]$. However, it is not clear whether this newly discovered particle is a pure SM Higgs boson, or whether it is part of an extension of the SM, which might also include multiple or composite Higgs-like states. Precise measurements of the Higgs couplings and branching ratios will be required to resolve this question. Another, more direct way to gain insights about physics beyond the SM (BSM) would obviously be to discover more new particles directly.

A well-motivated extension of the SM is the Minimal Supersymmetric SM (MSSM), a fully renormalisable theory of high predictive power. In this model, the $\mathrm{U}(1), \mathrm{SU}(2)$ and $\mathrm{SU}(3)$ gauge couplings can unify, and a dark matter candidate can be naturally accommodated. Furthermore, supersymmetry stabilises the Higgs potential with respect to quadratically divergent radiative corrections. In the MSSM the Higgs mass is no longer a free parameter but becomes strongly constrained.

If nature is supersymmetric at the electroweak scale, a large number of new superpartner mass parameters is introduced whose values depend on the unknown mechanism of supersymmetry breaking at high energies. This poses a challenge for any phenomenological analysis. Due to the large number of unknown parameters it is very difficult to make reliable predictions for SUSY background processes, and in many cases the worst background contributions for SUSY signals originate in fact from SUSY processes. 
The experiments at the LHC have not yet found any hints for supersymmetry in squark and gluino searches 3, 4, which points towards a rather heavy coloured superpartner spectrum with soft masses above a TeV. However, there are large regions in the MSSM parameter space which lead to a SM-like Higgs with $m_{h}=126 \mathrm{GeV}$, a light electroweak SUSY sector, and a rather heavy coloured SUSY sector beyond the current LHC discovery range. In general, if the electroweak sector is light enough, it should be possible to discover neutralinos and charginos via Drell-Yan production at the LHC [5, 6], and the LHC experiments have already put limits on the electroweak-ino parameter space under several simplified model assumptions 7. 8. However, in cases where these light states are very close in mass and their decay products are therefore very soft, it will be almost impossible to observe such processes at the LHC. An example is given by scenarios in which the higgsino mass parameter $\mu$ is much smaller than the electroweak gaugino masses $M_{1}$ and $M_{2}$ (current bounds require only $|\mu| \gtrsim 100 \mathrm{GeV}$ in order to avoid the limits from LEP chargino searches [9]). Then, the new light states are a chargino and two neutralinos which are higgsino-like and almost mass degenerate. The chargino and the heavier neutralino therefore decay only into extremely soft SM particles and the invisible lightest neutralino.

The most "natural" versions of the phenomenological MSSM which are still not ruled out are indeed characterised by higgsino masses of a few $100 \mathrm{GeV}$, together with sub- TeV third-generation squarks and a moderately heavy gluino, while the rest of the spectrum can be in the multi-TeV range [10. Models with light higgsinos are also attractive for cosmology, either in combination with gravitino dark matter or with a higgsino-like neutralino as a non-thermally produced lightest supersymmetric particle (LSP); see e.g. [11].

While the light higgsino scenario is extremely challenging for the LHC, it may well be observable in the clean environment at an $e^{+} e^{-}$Linear Collider, for instance, at the International Linear Collider (ILC) [12, 13, 14. At the ILC, the striking feature of a precisely known initial state allows to successfully apply the initialstate radiation (ISR) method, i.e. to select only those signal events that are accompanied by a sufficiently hard photon. In this context, polarised beams help to significantly enhance the signal cross section, at least in the chargino case.

The subject of the present work is the phenomenology of light quasi-degenerate higgsinos at the ILC. We study their production and decay, and investigate in how far one can derive the fundamental MSSM parameters without assuming any specific SUSY breaking scheme if only the light higgsinos are kinematically accessible. We study the processes $e^{+} e^{-} \rightarrow \widetilde{\chi}_{1}^{+} \widetilde{\chi}_{1}^{-}$and $e^{+} e^{-} \rightarrow \widetilde{\chi}_{1}^{0} \widetilde{\chi}_{2}^{0}$ at $\sqrt{s}=500 \mathrm{GeV}$ with polarised beams and initial-state radiation at the ILC. We simulate the detector response for two MSSM benchmark points with different $\mathcal{O}(1 \mathrm{GeV})$ mass splittings between the chargino and neutralinos, leading to different final state SM particles. The uncertainties on masses, mass differences and cross sections as determined by our simulation study are then used as inputs for a fit in order to reconstruct the MSSM parameters $M_{1}, M_{2}$, $\mu$ and $\tan \beta$. Our overall goal is to determine both how well the chargino and neutralino masses can be measured at the ILC, and what could be inferred from such a measurement about the underlying SUSY model.

In the following chapters we first give an overview of the theoretical arguments in favour of light higgsinos and the phenomenological aspects of such scenarios in the MSSM. The expected SM background will also be discussed, including beam conditions and detector capabilities at the ILC. In Section 3 we define two benchmark scenarios on which our study will be based and provide the details for the detector simulation. In Section 4 we discuss the expected experimental results at the ILC. In Section 5 we present our results on reconstructing the fundamental parameters and the precision we expect to achieve with our analysis strategy.

\section{Theoretical context and analysis strategy}

There are two main reasons why higgsinos are distinguished among the MSSM superpartners of the SM particles. First, the size of the higgsino mass parameter $\mu$ is not necessarily related to the scale of SUSY breaking, as it is allowed by unbroken supersymmetry (it is indeed the only dimensionful MSSM parameter with this property). It can therefore be generated independently of the soft supersymmetry breaking terms, and at leading order does not receive any soft-term-dependent radiative corrections in the evolution from the high scale. Second, $\mu$ enters the tree-level Higgs potential and is therefore directly connected to the 
electroweak scale. For instance, the tree-level $Z$ boson mass is given in terms of the Higgs soft masses $m_{H_{u}, H_{d}}^{2}$, the ratio $\tan \beta$ of Higgs vacuum expectation values, and $\mu$ as

$$
m_{Z}^{2}=2 \frac{m_{H_{u}}^{2} \tan ^{2} \beta-m_{H_{d}}^{2}}{1-\tan ^{2} \beta}-2|\mu|^{2} \approx-2\left(m_{H_{u}}^{2}+|\mu|^{2}\right) .
$$

To avoid finely tuned large cancellations among these parameters, it follows that $\mu$ should not be too far above the electroweak scale, even if some or all of the squark, slepton and gaugino masses are much larger. These two arguments are the main motivation for investigating models where the higgsinos are light, while the other MSSM superpartners may be much heavier.

\subsection{Light higgsinos from UV-scale models}

From a top-down perspective, light higgsinos are equally well motivated. For instance, they are generic in certain models of hybrid gauge-gravity mediation [15, 16, whose main features we will now briefly recapitulate. In gauge-mediated SUSY breaking, some of the hidden sector states carry SM gauge charges. These messenger fields acquire large supersymmetric masses $M$, and messenger loops generate soft terms of the order

$$
m_{\mathrm{soft}} \sim \frac{n}{16 \pi^{2}} \frac{F}{M}
$$

where $\sqrt{F}$ is the scale of SUSY breaking, and $n$ roughly counts the number of contributing messenger states. In addition to these, generically there are also gravity-mediated contributions to soft terms as well as a gravity-mediated effective $\mu$ parameter, generated by gravitationally suppressed interactions between the hidden sector and the MSSM. They are of the order of the gravitino mass,

$$
m_{3 / 2} \sim \mu \sim \frac{F}{M_{\text {Planck }}}
$$

In models where the MSSM is embedded in a more fundamental theory, for instance in superstring compactifications, the details of supersymmetry breaking are often unknown, while the spectrum of sub-Planckian states can still be calculated. In phenomenologically promising models, these typically consist of the MSSM along with a multitude of additional exotic states, some of which are charged under the SM gauge groups (see e.g. 17, 18, and references therein). These exotic fields can be given large masses and act as gauge mediation messengers. A natural scale for them to decouple is the scale of the Grand Unification,

$$
M \sim M_{\mathrm{GUT}}=2 \cdot 10^{16} \mathrm{GeV} \approx \frac{1}{16 \pi^{2}} M_{\text {Planck }}
$$

With some simple assumptions about the couplings of the messengers to the SUSY-breaking fields, and choosing parameters such that $m_{3 / 2}$ is of the order of the electroweak scale, one can obtain a realistic soft term spectrum. For large messenger multiplicities $n \gtrsim 10$, the soft terms are predominantly generated by gauge mediation and can be in the multi- $\mathrm{TeV}$ range, while $\mu$ is induced by gravity mediation and of the order of $100-200 \mathrm{GeV}$.

The benchmark points we are using for the present analysis are derived from such hybrid gauge-gravity mediation models with large messenger numbers. However, we stress that these are by no means the only possibility to obtain light higgsinos from high-scale models, and that our results on the light higgsino phenomenology are independent of a specific SUSY breaking model.

\subsection{Phenomenology}

In a more general context, scenarios with a rather heavy coloured sector and a mass-degenerate light chargino/neutralino sector can easily be constructed in the unconstrained MSSM, where the soft SUSYbreaking parameters are taken as independent. One simply chooses sufficiently large values for the squark and gluino masses (and possibly also for the slepton masses). 
At the tree level the chargino/higgsino sector [19] is determined by the $\mathrm{U}(1)$ bino mass parameter $M_{1}$, the $\mathrm{SU}(2)$ wino mass parameter $M_{2}$, the higgsino mass parameter $\mu$ and $\tan \beta$. The neutralino mass matrix in the $\left(\widetilde{B}, \widetilde{W}^{0}, \widetilde{H}_{d}^{0}, \widetilde{H}_{u}^{0}\right)$ basis is given by

$$
Y=\left(\begin{array}{cccc}
M_{1} & 0 & -m_{Z} \cos \beta \sin \theta_{W} & m_{Z} \sin \beta \sin \theta_{W} \\
0 & M_{2} & m_{Z} \cos \beta \cos \theta_{W} & -m_{Z} \sin \beta \cos \theta_{W} \\
-m_{Z} \cos \beta \sin \theta_{W} & m_{Z} \cos \beta \cos \theta_{W} & 0 & -\mu \\
m_{Z} \sin \beta \sin \theta_{W} & -m_{Z} \sin \beta \cos \theta_{W} & -\mu & 0
\end{array}\right) .
$$

Since $Y$ is complex symmetric, it can be diagonalised by one unitary matrix $N$ via $\mathbf{M}_{\widetilde{\chi}^{0}}=N^{*} Y N^{\dagger}$ (where the matrix $\mathbf{M}_{\widetilde{\chi}^{0}}$ contains the neutralino masses $M_{\widetilde{\chi}_{i}^{0}}$ on the diagonal). The mixing matrix $N$ relates the neutralino mass eigenstates to the neutral gauge eigenstates. For full analytical solutions of the system see [20, 21].

The chargino mass matrix in the $\left(\widetilde{W}^{+}, \widetilde{H}^{+}\right)$basis,

$$
X=\left(\begin{array}{cc}
M_{2} & \sqrt{2} m_{Z} \cos \theta_{W} \sin \beta \\
\sqrt{2} m_{Z} \cos \theta_{W} \cos \beta & \mu
\end{array}\right),
$$

is not symmetric and therefore has to be diagonalised via the bi-unitary transformation $\mathbf{M}_{\tilde{\chi}^{+}}=U^{*} X V^{\dagger}$. $U$ and $V$ relate the chargino mass eigenstates to the charged gauge eigenstates. In general, $M_{1}, M_{2}$ and $\mu$ are complex-valued parameters. By a suitable rotation of the gaugino and higgsino fields, $M_{2}$ can be made real and positive without loss of generality. In the following we assume that also $M_{1}$ and $\mu$ are real (positive or negative) so no new sources of $\mathrm{CP}$ violation are introduced.

One can easily classify three limiting cases: in the case $\left|M_{1}\right|<M_{2} \ll|\mu|$, one obtains $\left|M_{1}\right| \approx M_{\widetilde{\chi}_{1}^{0}}$ with a bino-like $\widetilde{\chi}_{1}^{0}, M_{2} \approx M_{\widetilde{\chi}_{2}^{0}}, M_{\widetilde{\chi}_{1}^{ \pm}}$with wino-like $\widetilde{\chi}_{2}^{0}$ and $\widetilde{\chi}_{1}^{ \pm}$, and higgsino-like heaviest states with masses $|\mu| \approx M_{\widetilde{\chi}_{3,4}^{0}}, M_{\widetilde{\chi}_{2}^{ \pm}}$, cf. Eqs. (5) and (6). By contrast, the hierarchy $M_{2}<\left|M_{1}\right| \ll|\mu|$ leads to the lightest two states being mass degenerate and wino-like, $M_{2} \approx M_{\widetilde{\chi}_{1}^{0}}, M_{\widetilde{\chi}_{1}^{ \pm}}$but a bino-like $\widetilde{\chi}_{2}^{0}$ with mass $\left|M_{1}\right| \approx M_{\widetilde{\chi}_{2}^{0}}$, while $\widetilde{\chi}_{3,4}^{0}$ and $\widetilde{\chi}_{2}^{ \pm}$remain higgsino-like and heavy as before.

Choosing $|\mu| \ll\left|M_{1}\right|<M_{2}$ (or $|\mu| \ll M_{2}<\left|M_{1}\right|$ ) leads to mass-degenerate lightest states with $|\mu| \approx$ $M_{\widetilde{\chi}_{1,2}^{0}}, M_{\widetilde{\chi}_{1}^{ \pm}}$but $\left|M_{1}\right| \approx M_{\widetilde{\chi}_{3}^{0}}\left(\right.$ or $\left.\left|M_{1}\right| \approx M_{\widetilde{\chi}_{4}^{0}}\right)$ and $M_{2} \approx M_{\widetilde{\chi}_{2}^{ \pm}}, M_{\widetilde{\chi}_{4}^{0}}\left(\right.$ or $M_{2} \approx M_{\widetilde{\chi}_{2}^{ \pm}}, M_{\widetilde{\chi}_{3}^{0}}$ ). The lighter neutralinos and the lightest chargino are then mostly higgsino-like and have unsuppressed couplings to the SM gauge bosons. This is the scenario which we are investigating.

It is instructive to explicitly write down the tree-level masses of the three light states in the case that $M_{1}$ and $M_{2}$ are large. They are found by diagonalising the mass matrices of Eqs. (5) and (6), and given by

$$
\begin{aligned}
& M_{\widetilde{\chi}_{1,2}^{0}}=\eta_{1,2}\left(|\mu| \mp \frac{m_{Z}^{2}}{2}(1 \pm \sin 2 \beta \operatorname{sign}(\mu))\left(\frac{\sin ^{2} \theta_{W}}{M_{1}}+\frac{\cos ^{2} \theta_{W}}{M_{2}}\right)\right), \\
& M_{\widetilde{\chi}_{1}^{ \pm}}=|\mu|-\sin 2 \beta \operatorname{sign}(\mu) \cos ^{2} \theta_{W} \frac{m_{Z}^{2}}{M_{2}},
\end{aligned}
$$

up to terms suppressed by higher powers of $M_{i}$, see e.g. [22. The factors $\eta_{1,2}$ denote the sign of the mass eigenvalue, that depends on the CP-quantum number of the corresponding neutralino [19, 20].

The wino and bino admixtures are small, of the order $m_{Z} / M_{1,2}$. For instance, for $\mu>0$, the mass eigenstates are decomposed as

$$
\begin{aligned}
\chi_{1}^{0} & =\frac{1}{\sqrt{2}}\left(\widetilde{H}_{d}^{0}-\widetilde{H}_{u}^{0}\right)+\frac{\sin \beta+\cos \beta}{\sqrt{2}} \frac{m_{Z}}{M_{1}} \sin \theta_{W} \widetilde{B}-\frac{\sin \beta+\cos \beta}{\sqrt{2}} \frac{m_{Z}}{M_{2}} \cos \theta_{W} \widetilde{W}^{0}, \\
\chi_{2}^{0} & =\frac{1}{\sqrt{2}}\left(\widetilde{H}_{d}^{0}+\widetilde{H}_{u}^{0}\right)-\frac{|\sin \beta-\cos \beta|}{\sqrt{2}} \frac{m_{Z}}{M_{1}} \sin \theta_{W} \widetilde{B}+\frac{|\sin \beta-\cos \beta|}{\sqrt{2}} \frac{m_{Z}}{M_{2}} \cos \theta_{W} \widetilde{W}^{0}, \\
\chi_{1}^{+} & =\widetilde{H}_{u}^{+}-\sqrt{2} \sin \beta \frac{m_{W}}{M_{2}} \widetilde{W}^{+}, \\
\chi_{1}^{-} & =\widetilde{H}_{d}^{-}-\sqrt{2} \cos \beta \frac{m_{W}}{M_{2}} \widetilde{W}^{-},
\end{aligned}
$$


where we have only written the respective leading-order terms of the Weyl gaugino and higgsino components. For multi-TeV gaugino masses, the gaugino admixtures are of the order of a few percent, and the mass splittings are of the order of 1-10 GeV. Explicitly, the tree-level mass difference between the lightest neutralino and the lightest chargino reads, again for $\mu>0$,

$$
M_{\widetilde{\chi}_{1}^{ \pm}}-M_{\widetilde{\chi}_{1}^{0}}=\frac{m_{Z}^{2}}{2}\left[\sin 2 \beta\left(\frac{\sin ^{2} \theta_{W}}{M_{1}}-\frac{\cos ^{2} \theta_{W}}{M_{2}}\right)+\left(\frac{\sin ^{2} \theta_{W}}{M_{1}}+\frac{\cos ^{2} \theta_{W}}{M_{2}}\right)+\mathcal{O}\left(\frac{\mu}{M_{i}^{2}}\right)\right],
$$

which for $\tan \beta \gg 1$ further simplifies to

$$
M_{\widetilde{\chi}_{1}^{ \pm}}-M_{\widetilde{\chi}_{1}^{0}}=\frac{m_{Z}^{2}}{2}\left(\frac{\sin ^{2} \theta_{W}}{M_{1}}+\frac{\cos ^{2} \theta_{W}}{M_{2}}\right)+\mathcal{O}\left(\frac{\mu}{M_{i}^{2}}, \frac{1}{\tan \beta}\right) .
$$

For the neutralinos, the mass difference is

$$
M_{\widetilde{\chi}_{2}^{0}}-M_{\widetilde{\chi}_{1}^{0}}=m_{Z}^{2}\left(\frac{\sin ^{2} \theta_{W}}{M_{1}}+\frac{\cos ^{2} \theta_{W}}{M_{2}}\right)+\mathcal{O}\left(\frac{\mu}{M_{i}^{2}}\right) .
$$

Remarkably, it does not depend on $\tan \beta$ in the leading approximation. The corresponding expressions for $\mu<0$ are similar and the phenomenology of that parameter range has been discussed in 23. In our case the sign of $\mu$ has negligible impact on observables. Equations (7)-(11) hold for positive and negative values of $M_{1}$. It is interesting to note that negative values of $M_{1}$ allow for cancellations of wino and bino contributions to neutralino masses. Generally, they will result in different mass patterns depending on the sign of $M_{1}$. In particular, for low values of $\tan \beta$ the lightest neutralino can be heavier than the light chargino, i.e. $M_{\widetilde{\chi}_{1}^{ \pm}}<M_{\widetilde{\chi}_{1}^{0}}$. Both possibilities, $M_{1}>0$ and $M_{1}<0$, will be analysed in Section 5 .

Comparing with the analytical formulae of the neutralino (chargino) masses and couplings [21, 24, it is obvious that one can express the neutralino and chargino sector as functions of $\sin 2 \beta$ and $\cos 2 \beta$. In the large $\tan \beta$ limit, $|\cos 2 \beta| \rightarrow 1$ and $\sin 2 \beta \rightarrow 2 / \tan \beta$, therefore all neutralino and chargino masses as well as the cross sections are only weakly dependent on $\tan \beta$. This weak dependence is also reflected in our final results, cf. Section 5 .

Radiative corrections to the chargino and neutralino observables, see e.g. 25] (and references therein for a more complete list of studies), can be sizeable and increase the mass splittings by several hundreds of $\mathrm{MeV}$. These corrections will lift the degeneracy between higgsino states. If the tree-level mass difference is substantially less than a $\mathrm{GeV}$, a correction of $200 \mathrm{MeV}$ [26] will have a profound impact on the lifetime and decay branching ratios. This may also be exploited to better determine the underlying MSSM parameters, see [27. A detailed analysis of NLO corrections in this regard, is, however, beyond the scope of this study and in the following we will assume tree-level relations.

At the ILC higgsino production proceeds via $Z$ or $\gamma$ exchange in the $s$-channel, see Fig. 1 In general, $t$ and $u$-channel exchange of selectrons or sneutrinos is also possible, but in the higgsino case they are highly suppressed due to the small Yukawa couplings. Hence, even if the selectrons and sneutrinos were relatively light, they would not affect any observables. Higgsinos will be produced in $\widetilde{\chi}_{1}^{0} \widetilde{\chi}_{2}^{0}$ or $\widetilde{\chi}_{1}^{+} \widetilde{\chi}_{1}^{-}$pairs (the $Z \widetilde{\chi}_{1}^{0} \widetilde{\chi}_{1}^{0}$ and $Z \widetilde{\chi}_{2}^{0} \widetilde{\chi}_{2}^{0}$ couplings vanish in the pure higgsino limit) 1 Both $\widetilde{\chi}_{2}^{0}$ and $\tilde{\chi}_{1}^{ \pm}$will undergo three-body decays into $\widetilde{\chi}_{1}^{0}$ via off-shell $Z$ and $W$ bosons $\left[28\right.$. The decay $\widetilde{\chi}_{2}^{0} \rightarrow W^{ \pm *} \widetilde{\chi}_{1}^{\mp}$ is also possible but kinematically highly suppressed. The detector signature comprises exclusively a few low-energetic leptons or hadrons, whose softness poses a major challenge to experiment. For small mass splittings, the radiative decay $\widetilde{\chi}_{2}^{0} \rightarrow \widetilde{\chi}_{1}^{0} \gamma$ via a $W$-chargino loop can also become very important [29. More details on higgsino production and decay will be given below when we discuss the details of our simulation.

\subsection{Standard Model background}

For the discussion of the SM processes at the ILC it is convenient to classify them by the initial state and the number of final state fermions. Due to the very strong fields between the colliding bunches, there is a

\footnotetext{
${ }^{1}$ This is a consequence of the fact that $\widetilde{H}_{u}^{0}$ and $\widetilde{H}_{d}^{0}$ have opposite weak isospin, and that both $\widetilde{\chi}_{1}^{0}$ and $\widetilde{\chi}_{2}^{0}$ contain equally large $\widetilde{H}_{u}^{0}$ and $\widetilde{H}_{d}^{0}$ admixtures as seen from Eq. (8).
} 


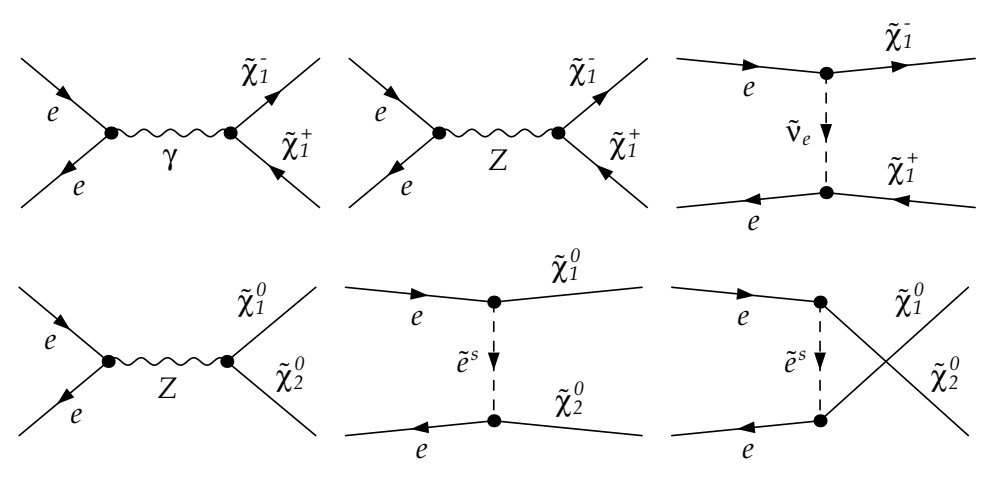

Figure 1: Chargino (upper row) and neutralino (lower row) production graphs at tree level. Note that $t$ - and $u$-channel selectron/sneutrino exchange graphs are negligible in our scenario due to a small Yukawa coupling between sleptons and higgsinos.

sizeable photon component from back-scattered synchrotron radiation in the ILC beams 31, 32. Therefore, the initial states can be $e^{+} e^{-}$, but also $\gamma \gamma$, or $\gamma e^{ \pm}$. For $e^{+} e^{-}$and $\gamma \gamma$, the final state has an even number of fermions: $2 \mathrm{f}, 4 \mathrm{f}, 6 \mathrm{f}, \ldots$, while the $\gamma e^{ \pm}$initial state gives rise to final states with an odd number of fermions: 1f, $3 \mathrm{f}, 5 \mathrm{f}, \ldots$ In this notation, $\gamma$ can represent both real beam photons or virtual ones. For initial virtual photons, the final state is understood to also contain the electron and/or positron that emitted the photon (the "beam-remnant"), in addition to the fermion(s).

The signal comprises events with a few low-energy charged particles and no other activity. Possible SM processes giving this topology are of two types. Either $(i)$ events with large amounts of energy in invisible neutrinos, or (ii) events where a large fraction of the energy escapes with particles going in un-instrumented regions of the detector; in an ILC detector, the only such regions are the holes in the low-angle calorimeter through which the beam pipes enter the detector.

The first background type mainly contains events with two $\tau \mathrm{s}$, possibly together with additional neutrinos, i.e. the subset of the classes $e^{+} e^{-} \rightarrow 2 \mathrm{f}$ and $e^{+} e^{-} \rightarrow 4 \mathrm{f}$ where one of the fermion pairs are $\tau$ leptons, and the other one (if present) is a neutrino pair. These channels do not have cross sections much larger than the signal. In addition, only a small fraction of the $\tau$ s will decay to the very soft visible system which is typical for the signal. Hence, while it is not negligible, this background type is expected to be manageable without too strong cuts on the event-topology.

The potentially most severe background processes are of type (ii), and are expected to be the high cross section multi-peripheral process $e^{+} e^{-} \rightarrow e^{+} e^{-} \gamma^{*} \gamma^{*} \rightarrow e^{+} e^{-} f \bar{f}$, i.e. the $\gamma \gamma \rightarrow 2 \mathrm{f}$ class. In such events, the final beam-remnant electrons and positrons carry nearly the full beam energy in most cases, have low transverse momentum, and therefore leave the detector unseen through the outgoing beam pipe. The $f \bar{f}$ pair, on the other hand, can be emitted at any angle, and tends to have low momentum. In other words, these events have a large resemblance to the signal. However, if one of the incoming electrons emits an ISR photon at an angle and energy high enough to be detected, this electron will recoil against the photon, changing its direction sufficiently to deflect it into the acceptance of the detector. In order to reduce this background to acceptable levels, we therefore require that the accepted signal candidate events should contain a detected ISR photon. Also the other component of the $\gamma \gamma \rightarrow 2 \mathrm{f}$ class, where the initial photons are real photons from the photon component of the beams, is suppressed by this requirement. This is simply due to the fact that, in this case, there are no initial charged particles that could emit an ISR photon.

Instead, the most severe remaining background is expected to arise from the $e \gamma \rightarrow 3 \mathrm{f}$ class, in particular from the $t$-channel diagram with incoming real photons. This process is similar to Compton scattering, with the difference that the scattered photon is virtual, and gives rise to an $f \bar{f}$-pair. In this case, the hard ISR photon does not recoil against the beam electron, but against the $f \bar{f}$-system, like in the signal events. However, in the event as a whole, a large amount of energy is carried away by the exchanged electron once it has been brought back on-shell by the beam-photon. This electron will receive most of the photon's energy, and closely follow its direction in the lab frame, i.e. along the beam pipe. As the second incoming particle 


\begin{tabular}{lccccccccc}
\hline & $n_{1}$ & $n_{2}$ & $n_{3}$ & $m_{\mathrm{GM}}$ & $m_{A}$ & $\tan \beta$ & $\mu^{\overline{\mathrm{DR}}}$ & $M_{1}^{\overline{\mathrm{DR}}}$ & $M_{2}^{\overline{\mathrm{DR}}}$ \\
\hline $\mathrm{dM} 1600$ & 20 & 28 & 11 & $190 \mathrm{GeV}$ & $2160 \mathrm{GeV}$ & 43.81 & $160 \mathrm{GeV}$ & $1.72 \mathrm{TeV}$ & $4.33 \mathrm{TeV}$ \\
$\mathrm{dM} 770$ & 46 & 46 & 20 & $250 \mathrm{GeV}$ & $4050 \mathrm{GeV}$ & 47.66 & $160 \mathrm{GeV}$ & $5.37 \mathrm{TeV}$ & $9.47 \mathrm{TeV}$ \\
\hline
\end{tabular}

Table 1: Input parameters and running gaugino masses for the benchmark points. Here $n_{1,2,3}$ refers to the messenger indices for $\mathrm{U}(1)_{Y}, \mathrm{SU}(2)_{L}$, and $\mathrm{SU}(3)_{C}$, respectively, $m_{\mathrm{GM}}$ is the characteristic gauge-mediated soft mass per messenger pair. $\mu \overline{\mathrm{DR}}$ is the running higgsino mass parameter in the $\overline{\mathrm{DR}}$ scheme evaluated at the soft mass scale (as defined e.g. in [33]); it generally differs from the on-shell $\mu$-parameter [34] by a few percent. Moreover, $m_{A}$ is the pole mass of the pseudoscalar Higgs, and $\tan \beta$ is the ratio of Higgs vacuum expectation values. For details about the precise definitions of $n_{i}$ and $m_{\mathrm{GM}}$ see $[16$. We also give the $\overline{\mathrm{DR}}$ values of $M_{1}$ and $M_{2}$ at the soft mass scale, which are determined at the high scale from the input parameters by the usual minimal gauge mediation relations.

is neutral in this case, no ISR will be emitted from it, and thus it cannot be deflected into the detector acceptance either.

\section{Benchmark points and simulation}

For our simulation we chose two benchmark points with the lightest Higgs mass which is compatible with the LHC discovery (within the theory uncertainty), namely 124 and $127 \mathrm{GeV}$. We will refer to them as dM1600 and dM770, according to their chargino-LSP mass difference. In the following sections we will discuss their low-scale spectrum and the higgsino production and decay modes at the ILC as well as their implementation into our simulation. We especially comment on the decay modes and branching ratios in the nearly mass-degenerate $\widetilde{\chi}_{1}^{ \pm}, \widetilde{\chi}_{1,2}^{0}$ system.

Beyond the two specific mass differences considered here, it has been shown recently that the ISR technique and the selection strategy described in the following are also sensitive in case of larger mass differences of a few GeV [30. As soon as the chargino-LSP mass difference becomes even larger, analyses without ISR requirement start to gain sensitivity and eventually take over. For mass differences smaller than the ones considered here, striking signatures like displaced vertices, kinks, anomalous ionisation etc. will make searches experimentally more easy. In this sense, the benchmarks chosen here fall into the experimentally most challenging regime.

\subsection{Spectrum generation, higgsino production and decay}

\section{Spectrum generation}

The general tree-level spectrum of our model was calculated using the spectrum generator SOFTSUSY [33]. The model we are considering is characterised by the high-scale parameters specified in Table 1. The parameters in the gaugino, squark, and slepton sector at the high scale are obtained from Eqs. (3) and (4) in [16, while the Higgs sector parameters are computed such that both the model predictions at the high scale are matched and electroweak symmetry breaking at the proper scale is obtained. With the input parameters in Table1. we obtain a spectrum which is mostly at the multi-TeV scale, with only the two lightest neutralinos as well as the light chargino masses within current collider ranges, and the SM-like Higgs boson with a mass within a $\pm 2 \mathrm{GeV}$ theory uncertainty interval from the LHC central value of $125.5 \mathrm{GeV}$.

The physical chargino and neutralino masses are calculated by SOFTSUSY using the running mass parameters $\mu^{\overline{\mathrm{DR}}}, M_{1}^{\overline{\mathrm{DR}}}$ and $M_{2}^{\overline{\mathrm{DR}}}$ as given in Table 1, which includes one-loop corrections [35]. The light chargino 
and neutralino spectrum, and the underlying on-shell $\mu$ and $M_{1,2}$ parameter: 2 are given by

$$
\begin{array}{ll}
\text { dM1600 } & M_{1}=1.70 \mathrm{TeV}, M_{2}=4.36 \mathrm{TeV}, \mu=165.89 \mathrm{GeV},\left.\tan \beta\right|_{m_{Z}}=44, \\
& M_{\widetilde{\chi}_{1}^{ \pm}}=165.77 \mathrm{GeV}, M_{\widetilde{\chi}_{1}^{0}}=164.17 \mathrm{GeV}, M_{\widetilde{\chi}_{2}^{0}}=166.87 \mathrm{GeV}, m_{h}=124 \mathrm{GeV} ; \\
\text { dM770 } & M_{1}=5.30 \mathrm{TeV}, M_{2}=9.51 \mathrm{TeV}, \mu=167.40 \mathrm{GeV},\left.\tan \beta\right|_{m_{Z}}=48, \\
& M_{\widetilde{\chi}_{1}^{ \pm}}=167.36 \mathrm{GeV}, M_{\widetilde{\chi}_{1}^{0}}=166.59 \mathrm{GeV}, M_{\widetilde{\chi}_{2}^{0}}=167.63 \mathrm{GeV}, m_{h}=127 \mathrm{GeV} .
\end{array}
$$

Note that the above masses include higher-order corrections, and therefore differ from the tree-level predictions in Eq. (7) at the sub-permil level 3 As stated before, the two lightest neutralinos are nearly completely higgsino-like, with negligible gaugino admixtures. The mass splittings are very small with $M_{\widetilde{\chi}_{1}^{ \pm}}-M_{\widetilde{\chi}_{1}^{0}}=770 \mathrm{MeV}(1.6 \mathrm{GeV})$ and $M_{\widetilde{\chi}_{2}^{0}}-M_{\widetilde{\chi}_{1}^{0}}=1.04 \mathrm{GeV}(2.7 \mathrm{GeV})$ in the dM770 (dM1600) scenario. This has interesting consequences especially for the decay, which we will discuss in the following.

\section{Production}

The production cross sections have been calculated with Whizard 1.95 [36] using the ILC set-up provided by the ILC Generator Group [37. It includes soft initial-state photon emission using an electron structure function [38] as well as the beam energy-spectra corresponding to the ILC Technical Design Report (ILC TDR) 39 .

Due to the small mass difference, only very few and soft visible particles will result from the higgsino decay. For reasons mentioned above, a realistic analysis will therefore need to require an additional photon from initial-state radiation to be seen in the detector. The ISR photon, in addition to its usefulness to suppress background, provides an elegant way to determine the $\widetilde{\chi}_{1}^{ \pm}$and $\widetilde{\chi}_{2}^{0}$ masses from their recoil against the photon. This has already been applied successfully for near-degenerate winos in AMSB scenarios [40.

We therefore determine the observable cross sections with one final state photon included in the hard matrix element. This photon is required to have a minimal invariant mass of $4 \mathrm{GeV}$ with the corresponding beam electron and an energy of more than $10 \mathrm{GeV}$, corresponding to the cut used later at the analysis stage. We have verified that the effect of double-counting with the soft initial-state photon emission is negligible.

Figure 2 shows the observable chargino and neutralino cross sections in the dM770 scenario as a function of the centre-of-mass energy, and for two different configurations of the polarisation of the beams, with the positron beam polarised to $-(+) 30 \%$, the electron beam to $+(-) 80 \%$ Figure 3 shows the same cross sections for a fixed centre-of-mass energy of $500 \mathrm{GeV}$ as a function of the positron polarisation. The chargino production can be strongly enhanced by choosing the appropriate beam helicities, whereas the differences are significantly smaller in the neutralino case. Beyond the signal enhancement, beam polarisation can be used to prove that the observed process is mediated purely by $s$-channel exchange of a $Z$-boson, i.e. by analysing the corresponding scaling factors between the polarised and unpolarised cross sections together with the order of magnitude of the cross section, see below and cf. discussion in 45. Effects from beam polarisation at an $e^{+} e^{-}$collider on chargino/neutralino pair-production have already been studied in [21, 24, 28, 5 and have successfully been exploited for full parameter determination in [46].

As mentioned, higgsinos are produced by $Z(\gamma, Z)$ exchange in the $s$-channel for neutralinos (charginos) respectively, cf. the diagrams shown in Fig. 1] It is then straightforward to explain the differences in production cross sections in Figs. 2 and 3 by considering only these diagrams. For neutralino production, the ratio of $\left(e_{R}^{+} e_{L}^{-} \rightarrow \widetilde{\chi}_{1}^{0} \widetilde{\chi}_{2}^{0}\right) /\left(e_{L}^{+} e_{R}^{-} \rightarrow \widetilde{\chi}_{1}^{0} \widetilde{\chi}_{2}^{0}\right) \sim 1.3$ for fully polarised beams can directly be obtained from

\footnotetext{
${ }^{2}$ The calculation of on-shell parameters follows Refs. 25] and 34. Three particles from the chargino/neutralino sector are chosen to be on-shell, e.g. $\widetilde{\chi}_{1}^{ \pm}, \widetilde{\chi}_{2}^{ \pm}$and $\widetilde{\chi}_{3}^{0}$. The most convenient choice should include "representative" states for each of the components, bino, wino and higgsino. This choice guarantees that the NLO corrections will be small [25]. Next, for fixed tan $\beta$ and using tree-level relations, see Eqs. (5) and (6), one calculates on-shell MSSM parameters. The masses of the remaining charginos and/or neutralinos will be shifted by appropriately calculated radiative corrections.

${ }^{3}$ These small differences in absolute masses, however, translate into sizeable corrections of the mass differences between the lightest chargino/second-lightest neutralino and the LSP. We accordingly correct for these higher-order contributions in the fit used for the determination of the fundamental parameters $M_{1}, M_{2}, \mu$, and $\tan \beta$; see Section 5 for further details.

${ }^{4}$ In the following, the notation $P\left(e^{+}, e^{-}\right)=(-30 \%,+80 \%)$ etc. will be used to denote the assumed beam polarisation.

${ }^{5}$ For (fully differential) chargino production at NLO, cf. [27, 41, 42, 43, 44].
} 

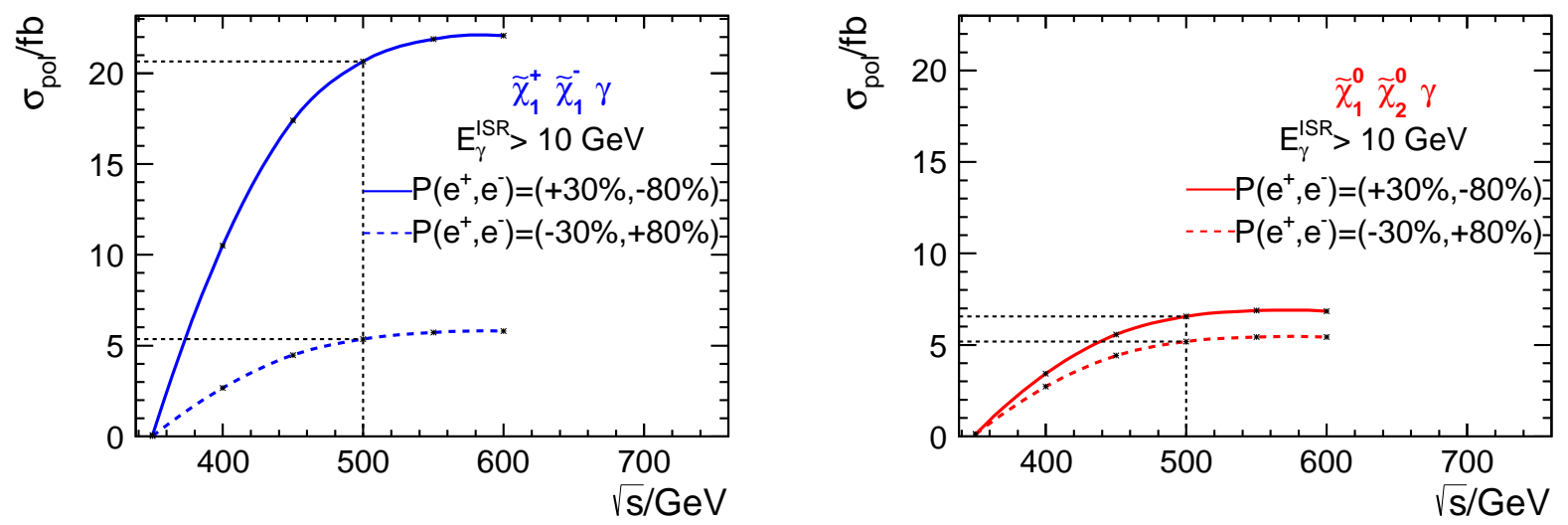

Figure 2: Chargino (left) and neutralino (right) production cross section in scenario dM770 for $P\left(e^{+}, e^{-}\right)=$ $(-30 \%,+80 \%)$ and $P\left(e^{+}, e^{-}\right)=(+30 \%,-80 \%)$ as a function of the centre-of-mass energy.
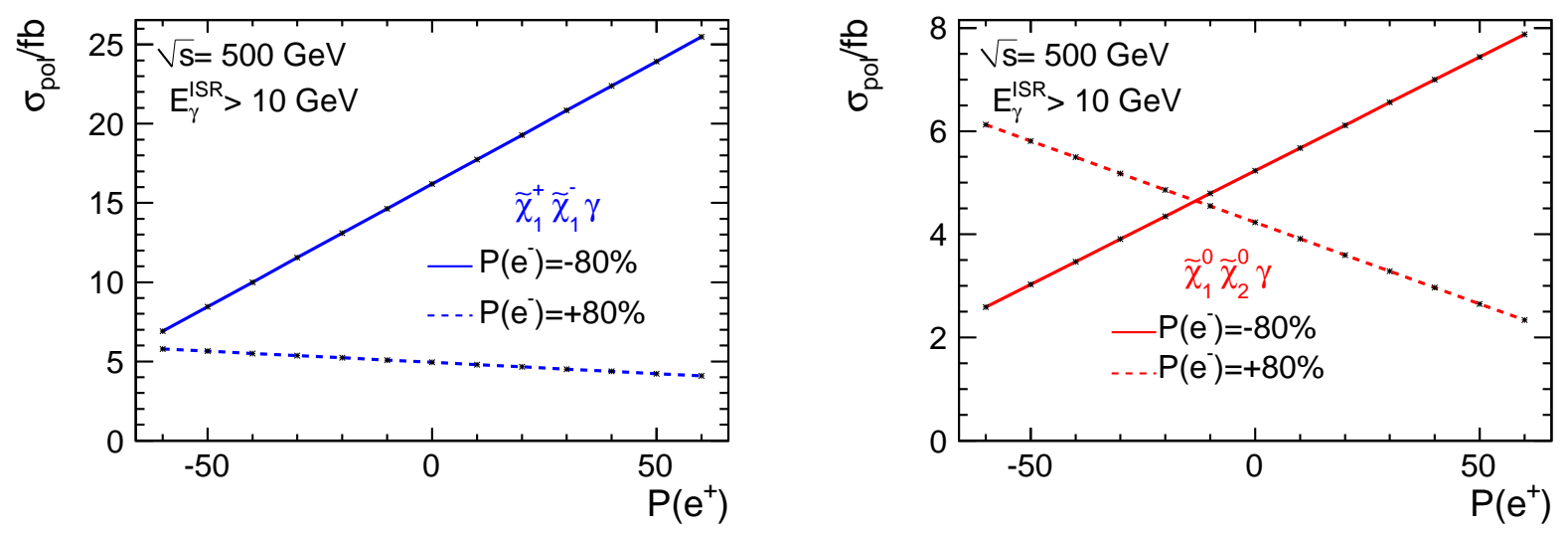

Figure 3: Chargino (left) and neutralino (right) production cross section in scenario dM770 at $\sqrt{s}=500 \mathrm{GeV}$ as a function of the positron polarisation for $P\left(e^{-}\right)=+80 \%$ and $P\left(e^{-}\right)=-80 \%$.

the ratio of the respective fermion couplings to the $Z$ boson, given by $L^{2} / R^{2}=\left[1-1 /\left(2 \sin ^{2} \theta_{W}\right)\right]^{2} \sim 1.3$, with $L=-\frac{1}{2}+\sin ^{2} \theta_{W}, R=\sin ^{2} \theta_{W}$. For arbitrarily polarised beams the scaling factor between polarised and unpolarised cross section is given by

$$
\sigma_{\text {pol }} / \sigma_{\text {unpol }}=\left(1+\frac{R^{2}-L^{2}}{R^{2}+L^{2}} P_{\text {eff }}\right) \cdot \mathcal{L}_{\text {eff }} / \mathcal{L}=\left(1-0.151 P_{\text {eff }}\right) \cdot \mathcal{L}_{\text {eff }} / \mathcal{L},
$$

where $\mathcal{L}_{\text {eff }}=\left(1-P\left(e^{-}\right) P\left(e^{+}\right)\right) \mathcal{L}$ denotes the normalised effective number of interactions and $P_{\text {eff }}=$ $\frac{P\left(e^{-}\right)-P\left(e^{+}\right)}{1-P\left(e^{-}\right) P\left(e^{+}\right)}$the effective polarisation (for details see [45]). It is expected to achieve high $\left|P_{\text {eff }}\right| \sim 95 \%$ and $\mathcal{L}_{\text {eff }} / \mathcal{L} \sim 1.5$ for centre-of-mass energies of $\sqrt{s} \geq 350 \mathrm{GeV}$ at the ILC [4] which leads to scaling factor of about $1.2(1.7)$ with $P\left(e^{-}, e^{+}\right)=(+80 \%,-60 \%)\left(P\left(e^{-}, e^{+}\right)=(-80 \%,+60 \%)\right)$ configuration of polarised $\left(e^{-}, e^{+}\right)$-beams.

In case of chargino pair production, the large difference in the cross sections stems from positive (in LR configuration) or negative (in RL configuration) interference of the $\gamma$ and $Z s$-channel contributions, which is again due to the structure of the fermion- $Z$ couplings. These characteristics (which are independent of additional photon radiation) are well reflected in the total cross sections displayed in Figures 2 and 3. 


\begin{tabular}{lcc}
\hline$\widetilde{\chi}_{1}^{+}$decay mode & $\mathrm{BR}(\mathrm{dM} 1600)$ & $\mathrm{BR}(\mathrm{dM} 770)$ \\
\hline$e \nu \widetilde{\chi}_{1}^{0}$ & $17.3 \%$ & $15.0 \%$ \\
$\mu \nu \widetilde{\chi}_{1}^{0}$ & $16.6 \%$ & $13.7 \%$ \\
$\pi^{+} \widetilde{\chi}_{1}^{0}$ & $16.5 \%$ & $60.4 \%$ \\
$\pi^{+} \pi^{0} \widetilde{\chi}_{1}^{0}$ & $28.5 \%$ & $7.3 \%$ \\
$\pi^{+} \pi^{0} \pi^{0} \widetilde{\chi}_{1}^{0}$ & $7.5 \%$ & $0.03 \%$ \\
$\pi^{+} \pi^{+} \pi^{-} \widetilde{\chi}_{1}^{0}$ & $7.1 \%$ & $0.03 \%$ \\
$\pi^{+} \pi^{+} \pi^{-} \pi^{0} \widetilde{\chi}_{1}^{0}$ & $2.4 \%$ & - \\
$\pi^{+} \pi^{0} \pi^{0} \pi^{0} \widetilde{\chi}_{1}^{0}$ & $0.5 \%$ & - \\
$K^{+} \widetilde{\chi}_{1}^{0}$ & $1.2 \%$ & $3.5 \%$ \\
$K^{0} \pi^{+} \widetilde{\chi}_{1}^{0}$ & $1.0 \%$ & $0.03 \%$ \\
$K^{+} \pi^{0} \widetilde{\chi}_{1}^{0}$ & $0.5 \%$ & $0.02 \%$ \\
\hline
\end{tabular}

Table 2: Chargino $\widetilde{\chi}_{1}^{+}$decay modes according to Herwig++ 2.6.0.

\begin{tabular}{lcc}
\hline$\widetilde{\chi}_{2}^{0}$ decay mode & $\mathrm{BR}(\mathrm{dM} 1600)$ & $\mathrm{BR}(\mathrm{dM} 770)$ \\
\hline$\gamma \widetilde{\chi}_{1}^{0}$ & $23.6 \%$ & $74.0 \%$ \\
$\nu \bar{\nu} \widetilde{\chi}_{1}^{0}$ & $21.9 \%$ & $9.7 \%$ \\
$e^{+} e^{-} \widetilde{\chi}_{1}^{0}$ & $3.7 \%$ & $1.6 \%$ \\
$\mu^{+} \mu^{-} \widetilde{\chi}_{1}^{0}$ & $3.7 \%$ & $1.5 \%$ \\
hadrons $+\widetilde{\chi}_{1}^{0}$ & $44.9 \%$ & $12.7 \%$ \\
\hline$\widetilde{\chi}_{1}^{ \pm}+X$ & $1.9 \%$ & $0.4 \%$ \\
\hline
\end{tabular}

Table 3: Neutralino $\widetilde{\chi}_{2}^{0}$ decay modes according to Herwig++ 2.6.0.

\section{Decay widths and branching ratios}

Due to the quite pronounced mass degeneracy in the $\widetilde{\chi}_{1}^{ \pm}, \widetilde{\chi}_{1}^{0}$ system, the $\widetilde{\chi}_{1}^{ \pm}$decays require a specific treatment, which we will now briefly describe. In case of small mass splittings, a perturbative treatment on parton level, followed by standard hadronisation, leads to large uncertainties for slight variations of the masses of the partonic decay products. In the scenarios considered here, this especially plays a role in the decays of the chargino, where the mass difference can be in the sub-GeV range. In fact, the same situation is encountered in the SM for the decay of $\tau \mathrm{s}$ into hadrons. In this case, a more appropriate description then follows an effective theory approach using hadronic currents. For the case of supersymmetry, such a description has been implemented in the event generator Herwig++ for $\widetilde{\chi}_{1}^{ \pm}$decays [48, 49. We therefore used Herwig++ to generate the according branching ratios and decays widths, they are given in Table 2 On the other hand, the branching ratios of the $\widetilde{\chi}_{2}^{0}$ are calculated at parton-level followed by hadronisation, with the results listed in Table $\sqrt{36}^{6}$ The decay widths of loop-induced processes have been cross checked with available analytic results [29]. They agree within $5 \%$ with the Herwig++ values.

\footnotetext{
${ }^{6}$ In principle, the mass difference for the $\widetilde{\chi}_{1}^{ \pm}$-LSP in dM1600 scenario and the $\widetilde{\chi}_{2}^{0}$-LSP in dM770 scenario are of similar order. However, since for neutral current decays there is no analogue to the charged current $\tau$-decay in the the SM, no dedicated code is yet available which treats the decays of $\widetilde{\chi}_{2}^{0}$ using hadronic currents. The effects of including such an improved description are in the line of future work.
} 


\subsection{Event generation and detector simulation}

\section{Event generation}

The simulation part of this study has been performed for a centre-of-mass energy of $\sqrt{s}=500 \mathrm{GeV}$. Signal events for the two benchmark scenarios have been generated with Whizard, using the same set-up as for the cross section calculation, described in Section 3.1. The charginos and neutralinos were generated by Whizard, with the subsequent decays simulated by Pythia [50]. The decay branching fractions used by Pythia were those given in Table 2 and 3 . As for the cross section calculation, one photon was included in the hard matrix element, and it was required to have a minimal invariant mass of $4 \mathrm{GeV}$ with the corresponding beam electron. The generator-level cut on the photon energy was, however, not applied in the event generation step, in order to be able to estimate the experimental acceptance after reconstruction.
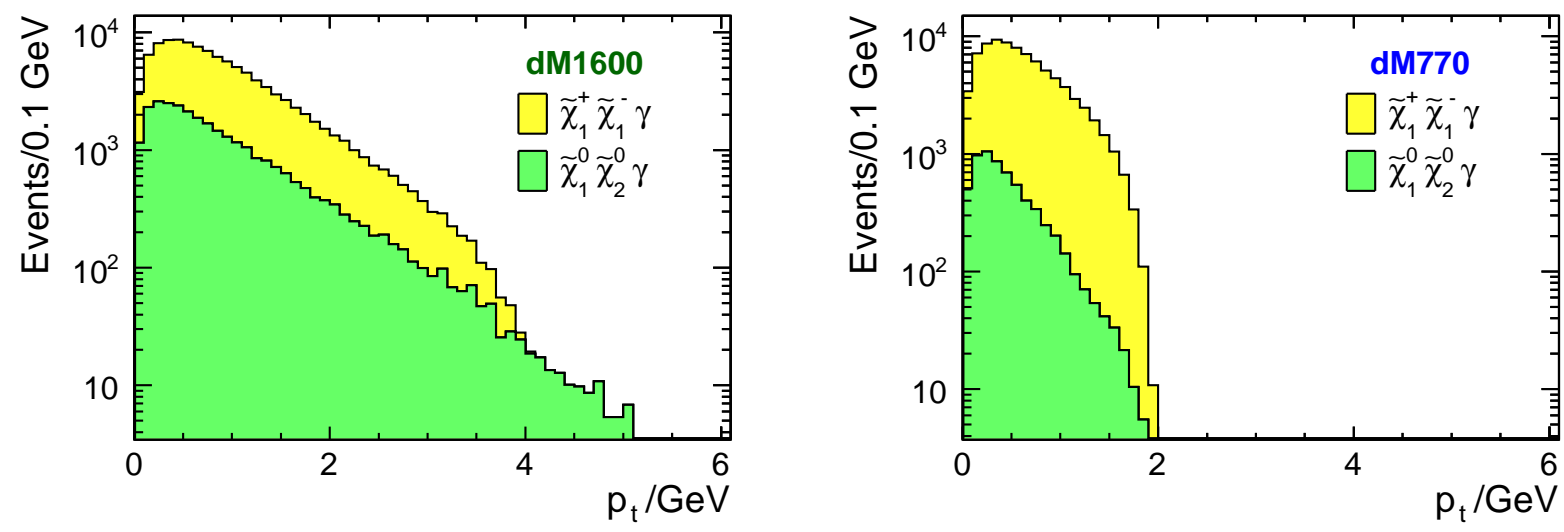

Figure 4: $p_{t}$ spectrum of visible chargino and neutralino decay products at generator level for the two scenarios. Left: dM1600, right: dM770.

Figure 4 shows the transverse momentum spectrum of the visible decay products of chargino and neutralino events at generator level for both benchmark scenarios. Especially in the dM770 scenario, the visible decay particles of the higgsinos are extremely soft, with $p_{t}<2 \mathrm{GeV}$. While for larger momenta the tracking at the ILC is almost 100\% efficient [51, the situation is more challenging for sub-GeV particles, especially due to the presence of random hits and additional tracks caused by $e^{+} e^{-}$-pair background from beamstrahlung. Therefore we will discuss the tracking efficiency in more detail in the following subsection.

The event samples centrally produced for the detector benchmarking in context of the ILC TDR 37. were used as SM background. Hence, the same Whizard version was used both for signal and background generation. Apart from some processes with very high cross sections, the available statistics corresponds at least to an integrated luminosity of $500 \mathrm{fb}^{-1}$. A notable exception was the $e \gamma \rightarrow 3 \mathrm{f}$ class, for which only an integrated luminosity of about $100 \mathrm{fb}^{-1}$ was available. This was taken care of by assigning appropriate weights to these events. Furthermore, radiative Bhabha events and pure photon final states deserve special comments in the context of this analysis. Radiative Bhabha events, i.e. $e^{+} e^{-} \rightarrow e^{+} e^{-} \gamma$, have been generated in a restricted phase space requiring an invariant mass of at least $4 \mathrm{GeV}$ between any two of the final state particles, a momentum transfer of $Q>4 \mathrm{GeV}$ between the incoming and outgoing electron (positron) and between the incoming lepton and the photon, resulting in a minimum acolinearity of the final state leptons. Pure photon final states have not been included in the production. However it has been shown in a previous full simulation study of a pure photon plus missing energy signature that the contribution after a very similar selection is negligible [52. Also not included are final states with only neutrinos and photons as well as QED Compton events. They could in principle contribute if overlaid with one of the low- $p_{t} \gamma \gamma \rightarrow$ hadrons events of which on average 1.2 occur per bunch-crossing for TDR machine parameters. However, it is rather improbable that such an overlay will mimic the exclusive decay modes of the higgsinos studied here. A quantitative estimate of the impact of overlay events is only meaningful in full, Geant4-based [53] simulation and thus beyond the scope of this study. 


\section{Detector simulation}

A detailed description of the ILD detector can be found in 51. The most important detector capabilities for this analysis comprise the tracking efficiency at very low momenta, the photon reconstruction and the hermeticity of the detector, including the ability to veto high energetic electrons in the forward calorimeters (LumiCal, LHCal and BeamCal). The tracking system is based on a Time Projection Chamber (TPC), augmented with silicon trackers on the inside, the outside and in the forward direction, as well as for vertexing. The transverse momentum resolution $\left(\Delta\left(1 / p_{t}\right)=\Delta\left(p_{t}\right) / p_{t}^{2}\right)$ is expected to be $2.0 \times 10^{-5} \mathrm{GeV}^{-1}$ asymptotically, worsening to $9.0 \times 10^{-5} \mathrm{GeV}^{-1}$ at $10 \mathrm{GeV}$, and to $9.0 \times 10^{-4} \mathrm{GeV}^{-1}$ at $1 \mathrm{GeV}$. In the lowangle region, charged particles will be efficiently detected down to $\theta=7^{\circ}(|\cos \theta|=0.993)$, while the only region not in the acceptance of the calorimetric system are the holes in the BeamCal for the beam pipes. Around the outgoing beam pipe, the radius of the hole is $20 \mathrm{~mm}$ at $z=3550 \mathrm{~mm}$, corresponding to $5.6 \mathrm{mrad}$. Since the crossing angle of the beams is $14 \mathrm{mrad}$ and the hole for the incoming beam pipe has $R=16 \mathrm{~mm}$, the lower edge of the acceptance increases to $18.5 \mathrm{mrad}$ at $\phi \approx 180^{\circ}$. The electromagnetic calorimeter (ECal) is a highly granular SiW sampling calorimeter with a transverse cell size of $5 \mathrm{~mm} \times 5 \mathrm{~mm}$ and 20 layers. In test-beam measurements with a prototype detector a resolution of $(16.6 \pm 0.1) / \sqrt{E(\mathrm{GeV})} \oplus(1.1 \pm 0.1) \%$ has been achieved [54].

We simulate the response of the ILD detector using the fast detector simulation SGV [55]. SGV derives the full covariance matrix of the track parameter reconstruction from the ILD detector geometry, taking into account point resolutions, positions of measurement planes and material effects like multiple scattering, bremsstrahlung and pair conversion. The calorimeter response has been parametrised to model the performance of the ILD particle flow reconstruction.

The response of the BeamCal is modelled taking into account the large energy depositions from beam backgrounds. The efficiency to tag an electron or photon on top of the beam background has been studied in full simulation and parametrised as function of the energy of the particle and the local background energy density at its impact point on the BeamCal surface.
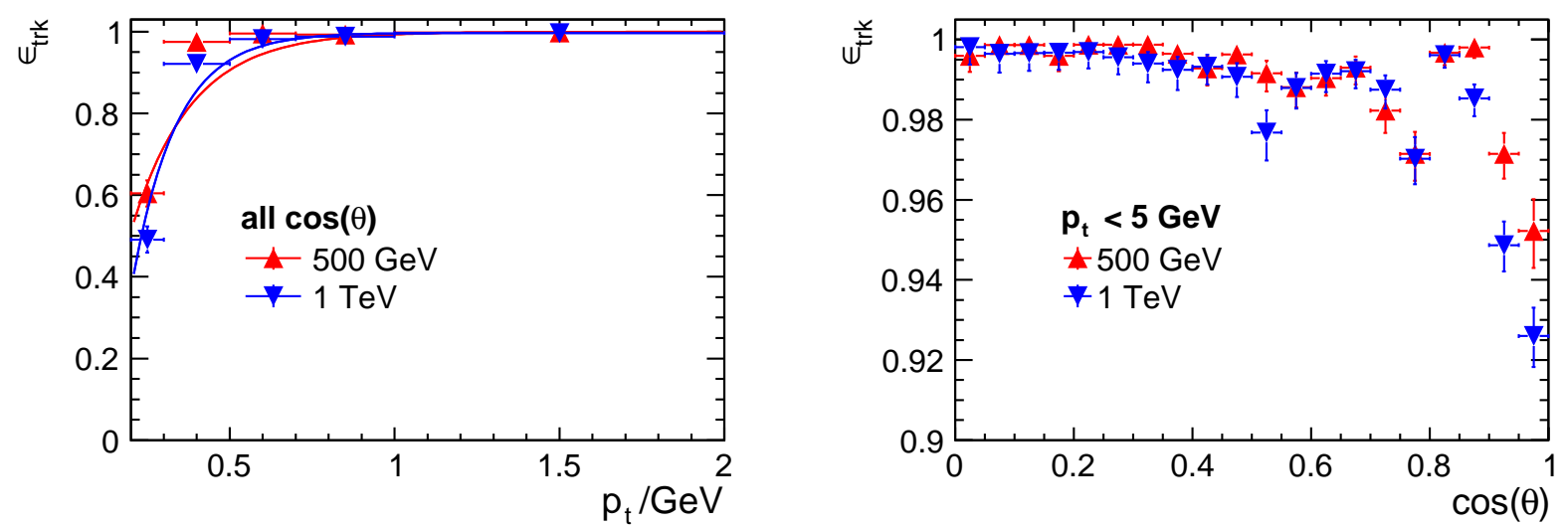

Figure 5: Tracking efficiency in $t \bar{t}$ events in the presence of pair background from beamstrahlung for $\sqrt{s}=500 \mathrm{GeV}$ and $\sqrt{s}=1 \mathrm{TeV}$, from full simulation of the ILD detector, assuming TDR beam parameters. Left: as a function of the transverse momentum $p_{t}$, Right: as a function of the absolute value of the cosine of the polar angle $|\cos \theta|$ for tracks with $p_{t}<5 \mathrm{GeV}$.

A special challenge for this analysis is the tiny mass gap, which leads to extremely low momenta of the visible higgsino decay products. Beyond what was done for the TDR studies, the tracking efficiency in the presence of $e^{+} e^{-}$pair background from beamstrahlung was determined in full simulation and reconstruction of the ILD detector. In order to have a conservative estimate, $e^{+} e^{-}$pairs generated with GuineaPig [56] were overlaid on events with much higher particle-multiplicity ( $t \bar{t}$ events) than what is expected from the signal studied here. These events were treated by Mokka [57, the full Geant4-based simulation of the ILD detector. For momenta larger than $1 \mathrm{GeV}$, track finding efficiency was found to be $99.5 \%$. For low- $p_{t}$ tracks, 
the track finding efficiency is shown as a function of $p_{t}$ and as function of $\cos \theta$ in Figure 5 . It can be seen that for beam-background conditions as expected at $\sqrt{s}=500 \mathrm{GeV}$, the tracking system is nearly fully efficient down to $p_{t}$ values around $400 \mathrm{MeV}$, dropping to about $60 \%$ for $p_{t}$ between 200 and $350 \mathrm{MeV} 7 \mathrm{For}$ all angles, the average tracking efficiency for all tracks with $p_{t}<5 \mathrm{GeV}$ was found to be larger than $99 \%$ in the barrel region, dropping slightly in the transition region between the barrel and the end-cap, but staying larger than $95 \%$ everywhere in the geometric acceptance of the tracking system.

In SGV, we implemented four parametrisations of the efficiency as function of $p_{t}$ for different polar angle ranges and applied it to the signal and the dominating background classes, namely the $e \gamma \rightarrow 3 \mathrm{f}$ and the $\gamma \gamma \rightarrow 2 \mathrm{f}$ processes, which are basically the only type of events remaining at the selection stage where the tracking efficiency for low-energetic particles enters.

\section{Experimental precision at the ILC}

In this section, we describe the event selection and the extraction of masses and cross sections in the two benchmark scenarios. All numbers refer to an integrated luminosity of $\int \mathcal{L} d t=500 \mathrm{fb}^{-1}$ each for two configurations of the beam polarisation, namely $P\left(e^{+}, e^{-}\right)=(+30 \%,-80 \%)$ and $P\left(e^{+}, e^{-}\right)=(-30 \%,+80 \%)$. With the current machine design, collecting $1 \mathrm{ab}^{-1}$ of data at $\sqrt{s}=500 \mathrm{GeV}$ takes 4 years of design ILC operation, based on typical machine availability times and efficiencies.

As a first step, a common preselection discriminating higgsino events against the majority of SM background processes is performed. Based on this, two different sets of cuts are applied for selecting either chargino or neutralino events and measuring the respective masses and cross sections.

\subsection{Preselection}

The aim of the preselection is to suppress the majority of SM background, while not yet discriminating charginos from neutralinos. The key features of the signal are that the events only contain a few lowenergetic but centrally produced decay products of the higgsinos, and the hard ISR photon. Thus, the following selection criteria are applied:

- There should be no significant activity in the BeamCal beyond the beam background in order to suppress events with high energetic electrons scattered under a small angle, e.g. from $\gamma \gamma$ and $e \gamma$ type of processes.

- The total number of reconstructed particles $N_{\mathrm{RP}}$ should be less than 15 .

- There should be exactly one reconstructed photon with $E_{\mathrm{ISR}}>10 \mathrm{GeV}$ and a polar angle $\theta_{\text {ISR }}$ fulfilling $\left|\cos \theta_{\text {ISR }}\right|<0.993$. The latter condition ensures that the photon is within the acceptance of the tracking system in order to be able to distinguish photons and electrons.

- Any other reconstructed particle in the event is required to be at least $20^{\circ}$ away from the beam axis, and thus within the acceptance of the TPC, and to have an energy $E_{\text {soft }}$ of less than $5 \mathrm{GeV} 8$

- Finally, the missing energy $E_{\text {miss }}$ should be larger than $300 \mathrm{GeV}$. This rejects the class of events where either the beam electron or positron is scattered under very small angles such that it escapes through the beam pipe with an energy of roughly $250 \mathrm{GeV}$.

- In order to ensure that the missing energy is not due to particles escaping along the beam pipe, the missing momentum vector is required to point well into the acceptance region of the detector by fulfilling $\left|\cos \theta_{\text {miss }}\right|<0.992$.

The resulting cut flow is displayed for $P\left(e^{+}, e^{-}\right)=(+30 \%,-80 \%)$ in Table 4 . At this stage of the selection, the SM background is reduced by four orders of magnitude and is dominated by $e \gamma \rightarrow 3 \mathrm{f}$ and

\footnotetext{
${ }^{7}$ For $p_{t}<200 \mathrm{MeV}$, the tracking efficiency has not been studied and therefore we set it to zero here.

${ }^{8}$ For charged particles, the energy is reconstructed from the momentum measured in the tracking system assuming the $\pi^{ \pm}$ mass.
} 
$\gamma \gamma \rightarrow 2 \mathrm{f}$ processes. The biggest loss in the signals is due to the ISR photon requirement, since at event generation only a minimal invariant mass of the photon with respect to the beam electron/positron of $4 \mathrm{GeV}$ was required, while the preselection demands a photon energy of at least $10 \mathrm{GeV}$, the latter being more stringent.

\begin{tabular}{lccccccc}
\hline & \multicolumn{2}{c}{$\mathrm{dM} 1600$} & \multicolumn{2}{c}{$\mathrm{dM770}$} & \multicolumn{3}{c}{ Standard Model } \\
\hline & $\tilde{\chi}_{1}^{+} \tilde{\chi}_{1}^{-} \gamma$ & $\tilde{\chi}_{1}^{0} \tilde{\chi}_{2}^{0} \gamma$ & $\tilde{\chi}_{1}^{+} \tilde{\chi}_{1}^{-} \gamma$ & $\tilde{\chi}_{1}^{0} \tilde{\chi}_{2}^{0} \gamma$ & $e e \rightarrow 2,4,6 \mathrm{f}$ & $e \gamma \rightarrow 3,5 \mathrm{f}$ & $\gamma \gamma \rightarrow 2,4 \mathrm{f}$ \\
\hline no cut & 38672 & 24250 & 38130 & 23940 & $2.6434 \times 10^{7}$ & $8.8820 \times 10^{7}$ & $9.7554 \times 10^{8}$ \\
BeamCal veto & 38591 & 24187 & 38054 & 23874 & $2.6284 \times 10^{7}$ & $8.8178 \times 10^{7}$ & $9.6757 \times 10^{8}$ \\
$N_{\mathrm{RP}}<15$ & 38591 & 24185 & 38054 & 23874 & $6.4968 \times 10^{6}$ & $6.5811 \times 10^{7}$ & $6.6308 \times 10^{8}$ \\
$N_{\text {ISR }}=1$ & 30058 & 9551 & 29675 & 9317 & $3.1640 \times 10^{6}$ & $1.5074 \times 10^{7}$ & $1.7752 \times 10^{7}$ \\
$\left|\cos \theta_{\text {soft }}\right|<0.9397$ & 21501 & 7318 & 23117 & 7458 & $7.1453 \times 10^{5}$ & $4.5646 \times 10^{6}$ & $4.7083 \times 10^{6}$ \\
$E_{\text {soft }}<5 \mathrm{GeV}$ & 20611 & 6615 & 22156 & 7110 & 9092 & $5.9732 \times 10^{5}$ & $1.2390 \times 10^{6}$ \\
$E_{\text {miss }}>300 \mathrm{GeV}$ & 20611 & 6615 & 22156 & 7110 & 6462 & $1.5822 \times 10^{5}$ & $4.6306 \times 10^{5}$ \\
$\left|\cos \theta_{\text {miss }}\right|<0.992$ & 19872 & 6365 & 21558 & 6872 & 5731 & $1.1837 \times 10^{5}$ & $3.3051 \times 10^{5}$ \\
\hline
\end{tabular}

Table 4: Cut-flow table of the preselection for an integrated luminosity of $500 \mathrm{fb}^{-1}$ and $P\left(e^{+}, e^{-}\right)=$ $(+30 \%,-80 \%)$.

\subsection{Chargino selection}

In order to discriminate chargino-pair events from the associated neutralino production, we select semileptonic events, i.e. events with one chargino decaying leptonically and the other hadronically. This signature does not appear in the associated neutralino production, since the $\widetilde{\chi}_{2}^{0}$ decays via a virtual $Z$ boson and thus either fully leptonically or fully hadronically. The main background contributions to the semi-leptonic signature are $\tau$-pairs, either from $e^{+} e^{-} \rightarrow \tau^{+} \tau^{-}$, from $e^{ \pm} \gamma \rightarrow e^{ \pm} \tau^{+} \tau^{-}$or from $\gamma \gamma \rightarrow \tau^{+} \tau^{-}$.

The cleanest semi-leptonic signature arises when the hadronic chargino decays to a single charged pion. In the dM770 scenario, this signature occurs in as many as 35\% of the chargino-pair events, cf. Table 2 . In the dM1600 scenario, the branching ratio to single pion is significantly lower due to the larger mass difference, so that only $11 \%$ of the chargino pairs give the semi-leptonic single pion signature. Therefore the $\pi^{ \pm} \pi^{0}$ channel is included for the dM1600 scenario by allowing the charged pion to be accompanied by two photons, raising the fraction of events with the target signature to 30\%. For the dM770 scenario, the gain in signal is too small to balance the penalty of larger background, and thus only the single pion mode is allowed in that scenario.

A very discriminative variable against $\tau$-events can be constructed from hadronic chargino decay:

$$
E_{\pi}^{*}=\frac{\left(\sqrt{s}-E_{\gamma}\right) E_{\pi}+\vec{p}_{\pi} \cdot \vec{p}_{\gamma}}{\sqrt{s^{\prime}}} .
$$

The reduced centre-of-mass energy $\sqrt{s^{\prime}}$ of the system recoiling against the ISR photon is defined as $s^{\prime}=$ $s-2 \sqrt{s} E_{\gamma}$, where $\sqrt{s}=500 \mathrm{GeV}$ is the nominal centre-of-mass energy. $E_{\pi}$ and $\vec{p}_{\pi}$ are the energy and 3 -momentum of the pion in the laboratory frame, while $\vec{p}_{\gamma}$ is the 3-momentum of the ISR photon in that frame. $E_{\pi}^{*}$ is related to the energy of the pion(s) in the rest frame of the chargino pair, which is only a few $\mathrm{GeV}$ for pions from chargino decays, but large for $\tau$ decays 9 Therefore, we require $E_{\pi}^{*}<3 \mathrm{GeV}$. As can be seen from Table 5, this cut is fully efficient in the dM770 scenario, but not in the dM1600 scenario. The reason is that the values of $E_{\pi}^{*}$ are closely related to the mass difference between the chargino and the LSP, which is nearly twice as large in the dM1600 scenario. However the losses occur in the less relevant

\footnotetext{
${ }^{9}$ In case of the additional $\pi^{0}$, the reconstructed four-momenta of the two photons are added to the charged pion fourmomentum.
} 
regime far from the endpoint. At this stage, the background is dominated by contributions from $\gamma \gamma$ events, in particular $\gamma \gamma \rightarrow \tau^{+} \tau^{-}$, which are typically back-to-back in the transverse plane and have large $\sqrt{s^{\prime}}$. Therefore the combined requirement of $\Phi_{\text {acop }}<2$ or $\sqrt{s^{\prime}}<480 \mathrm{GeV}$, where $\Phi_{\text {acop }}$ is the acoplanarity angle between the leptonic and the hadronic system, reduced this background by a factor of four.

\begin{tabular}{|c|c|c|c|c|c|}
\hline & \multicolumn{2}{|c|}{ dM1600 } & \multicolumn{3}{|c|}{ Standard Model } \\
\hline & $\tilde{\chi}_{1}^{+} \tilde{\chi}_{1}^{-} \gamma$ & $\widetilde{\chi}_{1}^{0} \widetilde{\chi}_{2}^{0} \gamma$ & $e e \rightarrow 2,4,6 \mathrm{f}$ & $e \gamma \rightarrow 3,5 \mathrm{f}$ & $\gamma \gamma \rightarrow 2,4 \mathrm{f}$ \\
\hline after preselection & 19872 & 6365 & 5731 & $1.1837 \times 10^{5}$ & $3.3051 \times 10^{5}$ \\
\hline$l^{ \pm} \pi^{ \pm}\left(\pi^{0}\right)$ & 5509 & 134 & 38 & 6197 & 13991 \\
\hline$E_{\pi}^{*}<3 \mathrm{GeV}$ & 4435 & 103 & 0 & 2635 & 6162 \\
\hline$\Phi_{\text {acop }}<2$ or $\sqrt{s^{\prime}}<480 \mathrm{GeV}$ & 3813 & 97 & 0 & 2564 & 1452 \\
\hline \multirow[t]{3}{*}{$E_{\text {miss }}>350 \mathrm{GeV}$} & 3812 & 97 & 0 & 1016 & 511 \\
\hline & \multicolumn{2}{|c|}{ dM770 } & \multicolumn{3}{|c|}{ Standard Model } \\
\hline & $\tilde{\chi}_{1}^{+} \tilde{\chi}_{1}^{-} \gamma$ & $\tilde{\chi}_{1}^{0} \widetilde{\chi}_{2}^{0} \gamma$ & $e e \rightarrow 2,4,6 \mathrm{f}$ & $e \gamma \rightarrow 3,5 \mathrm{f}$ & $\gamma \gamma \rightarrow 2,4 \mathrm{f}$ \\
\hline after preselection & 21558 & 6872 & 5731 & $1.1837 \times 10^{5}$ & $3.3051 \times 10^{5}$ \\
\hline$l^{ \pm} \pi^{ \pm}$ & 5489 & 38 & 19 & 2478 & 6754 \\
\hline$E_{\pi}^{*}<3 \mathrm{GeV}$ & 5489 & 38 & 0 & 1465 & 4755 \\
\hline$\Phi_{\text {acop }}<2$ or $\sqrt{s^{\prime}}<480 \mathrm{GeV}$ & 4600 & 36 & 0 & 1417 & 782 \\
\hline$E_{\mathrm{miss}}>350 \mathrm{GeV}$ & 4599 & 36 & 0 & 536 & 218 \\
\hline
\end{tabular}

Table 5: Cut-flow tables of the chargino selection in the two scenarios for an integrated luminosity of $500 \mathrm{fb}^{-1}$ and $P\left(e^{+}, e^{-}\right)=(+30 \%,-80 \%)$ following the preselection (cf. Tab. 44). For the dM1600 scenario, also the two-pion decay mode is included, therefore also the SM expectation changes. The chargino mass measurement is performed after the cut on the acoplanarity angle and the reduced centre-of-mass energy in both scenarios. The respective last line is applied for the cross section measurement and the determination of the mass difference.

Table 5 shows that at this level only a very small contribution from $\widetilde{\chi}_{1}^{0} \widetilde{\chi}_{2}^{0}$ events remains in the sample. This is important since we can assume the ability to model electroweak SM processes to any necessary precision by the time an ILC is actually running, whereas background contributions from other new physics processes, in our case the neutralino production, are a priori unknown.

\section{Chargino mass reconstruction}

The chargino mass is reconstructed using the reduced centre-of-mass energy, $\sqrt{s^{\prime}}$, introduced above. At the threshold, where the value of $s^{\prime}$ is denoted $\left.s^{\prime}\right|_{\text {thresh }}$, the chargino pair is produced nearly at rest, and $\sqrt{\left.s^{\prime}\right|_{\text {thresh }}}$ is twice the chargino mass, thus:

$$
M_{\widetilde{\chi}_{1}^{ \pm}}=\frac{1}{2} \sqrt{\left.s^{\prime}\right|_{\text {thresh }}}=\frac{1}{2} \sqrt{s-2 \sqrt{s} E_{\gamma}} .
$$

Figure 6 shows the resulting $\sqrt{s^{\prime}}$ distributions for both scenarios for an integrated luminosity of $500 \mathrm{fb}^{-1}$ with $P\left(e^{+}, e^{-}\right)=(+30 \%,-80 \%)$. The onset of the signal is clearly visible on top of the SM background, which stretches out to lower $\sqrt{s^{\prime}}$ values. The cut-off in the SM background near $\sqrt{s^{\prime}}=230 \mathrm{GeV}$ is due to the cut on $E_{\text {miss }}>300 \mathrm{GeV}$. This is chosen on purpose so that a signal-free region is available to fix the background level, here by fitting an exponential function with two free parameters $f(x)=p_{1} \cdot e^{-p_{2} \cdot x}$ to the SM prediction only (blue line). In a second step, a straight line is added on top of the background to 
model the signal contribution and fitted to the simulated data in the endpoint region (red line). The two parameters of the SM background function are fixed to the values obtained from the SM-only fit in the wider $\sqrt{s^{\prime}}$ window. It has been verified that the results are stable against reasonable variations of the fit ranges or the bounds on the background parameters. The chargino mass is fitted to $168.0 \pm 1.4 \mathrm{GeV}$ in the dM1600 scenario and to $168.6 \pm 1.0 \mathrm{GeV}$ in the dM770 case.
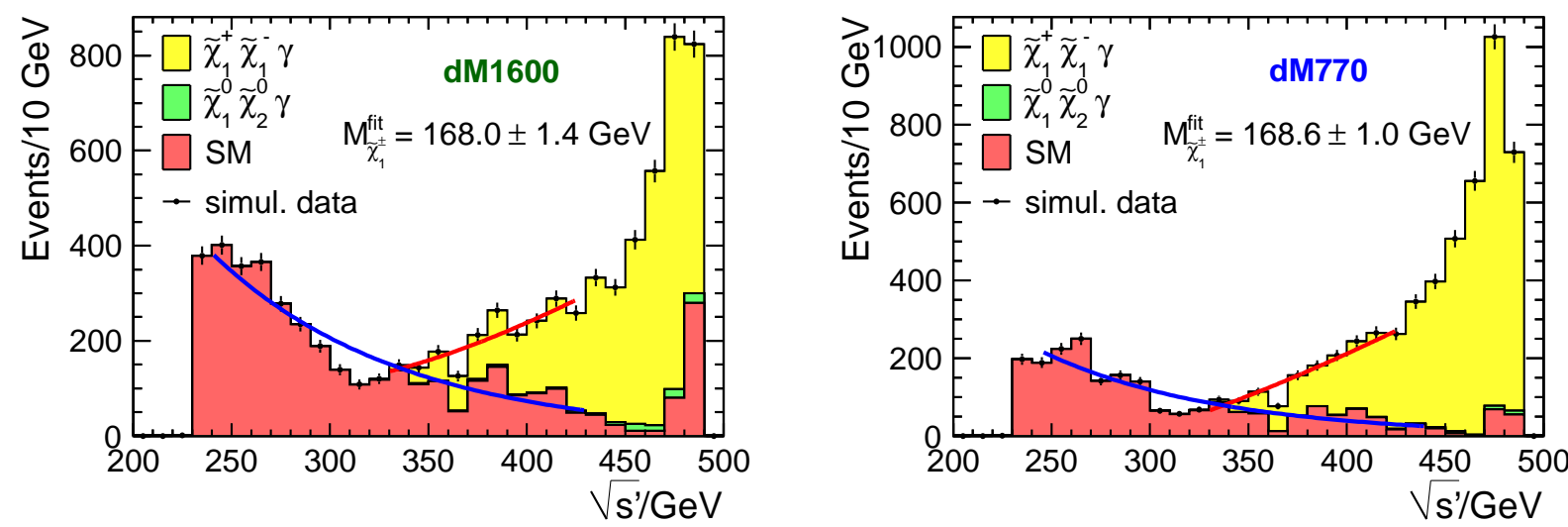

Figure 6: Distribution of the reduced centre-of-mass energy $\left(\sqrt{s^{\prime}}\right)$ of the system recoiling against the hard ISR photon for all events passing the chargino selection for an integrated luminosity of $500 \mathrm{fb}^{-1}$ with $P\left(e^{+}, e^{-}\right)=$ $(+30 \%,-80 \%) \cdot M_{\widetilde{\chi}_{1}^{ \pm}}$is determined from a linear fit to the distribution near the endpoint. Left: dM1600 scenario; Right: dM770 scenario.

The fitted central values agree within 1.6 (1.2) standard deviations with the respective input masses of $M_{\widetilde{\chi}_{1}^{ \pm}}=165.77 \mathrm{GeV}\left(M_{\widetilde{\chi}_{1}^{ \pm}}=167.36 \mathrm{GeV}\right)$ in the dM1600 (dM770) scenario. Since the relation between $\sqrt{s^{\prime}}$ and the chargino mass is only approximate, e.g. due to the approximation of equal chargino energies, but even more so due to the beam energy-spectrum, an exact agreement is not necessarily expected. Therefore we investigated the dependence of the fitted mass on the input mass by simulating signal samples with different chargino masses. In order to minimise a possible bias due to changes in the acceptance, all higgsino masses were varied simultaneously, so that e.g. the momentum distribution of the decay products does not change significantly.

Figure 7 shows the fitted mass as function of the true mass for both scenarios. They clearly display a linear behaviour, which can easily be used to calibrate the reconstruction method. The calibrated mass (and its uncertainty) can be found on the $x$-axis as a projection of the fitted values on the $y$-axis as indicated by the lines 10

At a first sight it might appear worrisome that both scenarios have different correlations between fitted and true masses. However one needs to remember that in the dM1600 case, an additional decay channel is included and thus different selections are applied in the two scenarios, which in this case changes the behaviour of the reconstruction. A substantial component of the uncertainty is correlated between all masses. This is due to the remaining background, and is most visible in the dM770 scenario, where the signal rate is higher and thus statistical fluctuations between the different signal samples at the different masses are smaller than in the dM1600 case. In the absence of any background, the statistical error on an individual mass determination would shrink to about $0.5 \mathrm{GeV}$.

After applying the calibration, we find an excellent agreement with the input mass values, at the price of the uncertainties increasing by $\sim 50 \%$ :

$$
\begin{array}{rlll}
\operatorname{dM1600} & : & M_{\widetilde{\chi}_{1}^{ \pm}}^{\text {cal }}=166.2 \pm 2.0 \mathrm{GeV} & \left(M_{\widetilde{\chi}_{1}^{ \pm}}^{\text {true }}=165.8 \mathrm{GeV}\right) \\
\operatorname{dM770} & : & M_{\widetilde{\chi}_{1}^{ \pm}}^{\text {cal }}=167.3 \pm 1.5 \mathrm{GeV} & \left(M_{\widetilde{\chi}_{1}^{ \pm}}^{\text {true }}=167.4 \mathrm{GeV}\right) .
\end{array}
$$

\footnotetext{
${ }^{10}$ The uncertainty on the calibration curve itself is not propagated to the final result, since its origin, namely the limited amount of available MC statistics especially for the SM background, will not be an issue in a real ILC measurement.
} 

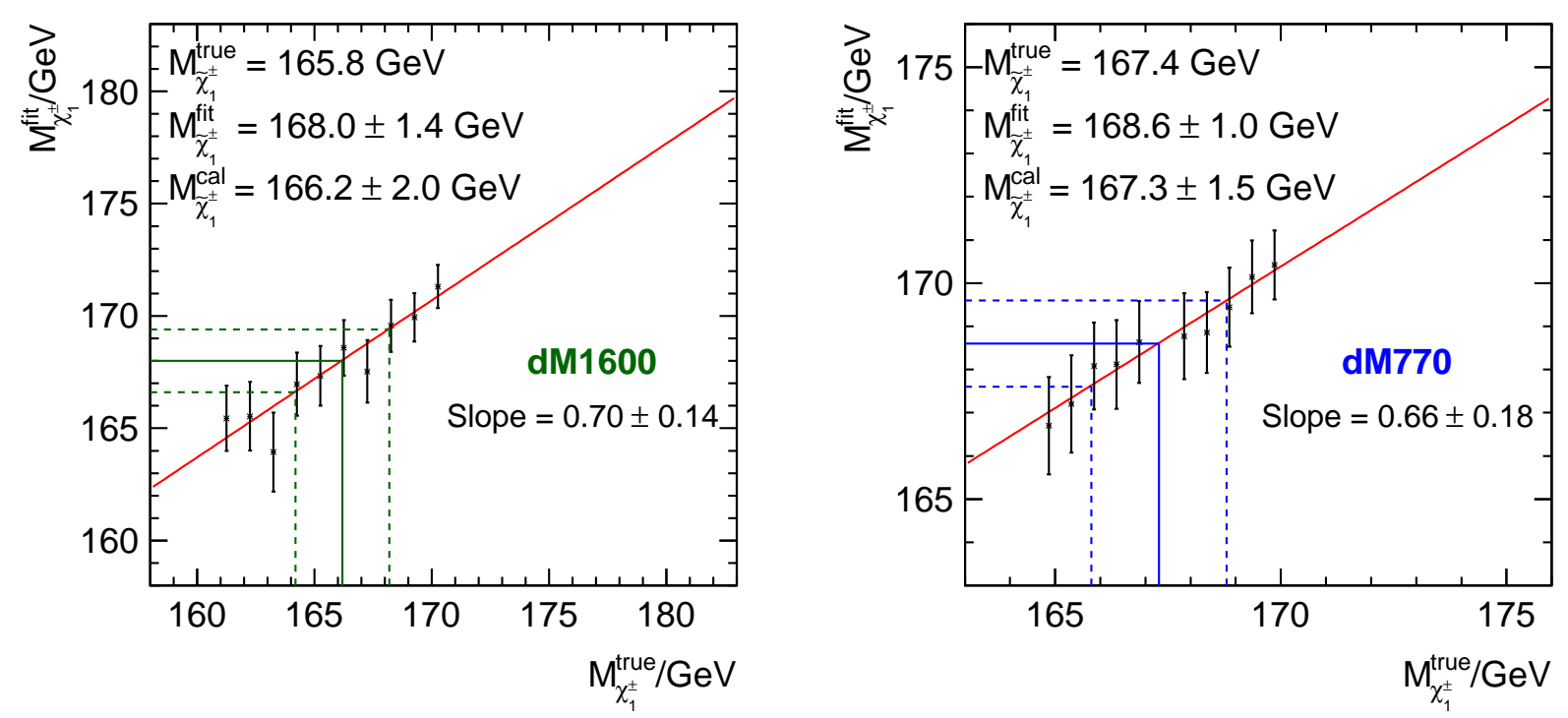

Figure 7: Calibration of the $\sqrt{s^{\prime}}$ method for the chargino mass determination. Left: dM1600 scenario; Right: dM770 scenario.

\section{Reconstruction of the chargino-LSP mass difference}

After a clear observation of the signal in the $\sqrt{s^{\prime}}$ spectrum and the determination of the chargino mass from the endpoint, the background can be further reduced by tightening the missing energy cut to $E_{\text {miss }}>$ $350 \mathrm{GeV}$. The resulting event count is displayed for both scenarios in the respective last line of Table 5

The mass difference between the chargino and the LSP is very small in both scenarios, namely $1.6 \mathrm{GeV}$ $(770 \mathrm{MeV})$ in the dM1600 (dM770) scenario. It is nevertheless accessible via the energy of the visible chargino decay products in the chargino-pair rest frame, calculated according to the definition in Eq. (15). If the charginos are actually produced at rest, $E_{\pi}^{*}$ can only take one value, viz:

$$
E_{\pi}^{*}=\frac{\left(M_{\widetilde{\chi}_{1}^{ \pm}}-M_{\widetilde{\chi}_{1}^{0}}\right)\left(M_{\widetilde{\chi}_{1}^{ \pm}}+M_{\widetilde{\chi}_{1}^{0}}\right)+m_{\pi}^{2}}{2 M_{\widetilde{\chi}_{1}^{ \pm}}}=\frac{\Delta(M) \Sigma(M)+m_{\pi}^{2}}{2 M_{\widetilde{\chi}_{1}^{ \pm}}}=\frac{1}{1 / \Delta(M)+1 / \Sigma(M)}+\frac{m_{\pi}^{2}}{2 M_{\widetilde{\chi}_{1}^{ \pm}}},
$$

wherein $\Delta(M)$ and $\Sigma(M)$ are the difference and sum of the chargino and the LSP masses, respectively. As both the mass difference and $m_{\pi}$ are several orders of magnitude smaller than the higgsino masses, $E_{\pi}^{*}$ is equal to the mass difference itself to a very good approximation. This is illustrated by the upper row of plots in Figure 8, which show the selected events in the $E_{\pi}^{*}-\sqrt{s^{\prime}}$ plane for both scenarios. The signal events form a distinctive triangular region, with a tip just at the mass difference indicated by the blue arrow. We select the tip of the triangle by requiring $\sqrt{s^{\prime}}<345 \mathrm{GeV}$, as indicated by the blue line, and then determine the tip position from the projection of the remaining events onto the $E_{\pi}^{*}$ axis. In a more complex analysis the whole two-dimensional $\sqrt{s^{\prime}}$ distribution could be exploited to determine the mass difference, potentially even together with the cross section.

The resulting $E_{\pi}^{*}$ distributions are displayed in the lower row of Figure 8 , again for both scenarios. The mass difference is then reconstructed as the mean of a Gaussian fitted to the distribution. Due to the limited amount of available SM events in the simulation, we subtract the SM background, assuming that precise theoretical predictions and sufficient MC statistics would be available for a real ILC measurement. However, the error bars include the full expected statistical fluctuations from the background. Especially in the case of the dM1600 scenario, the remaining signal is rather small, but still has a significance of more than $5 \sigma$. 

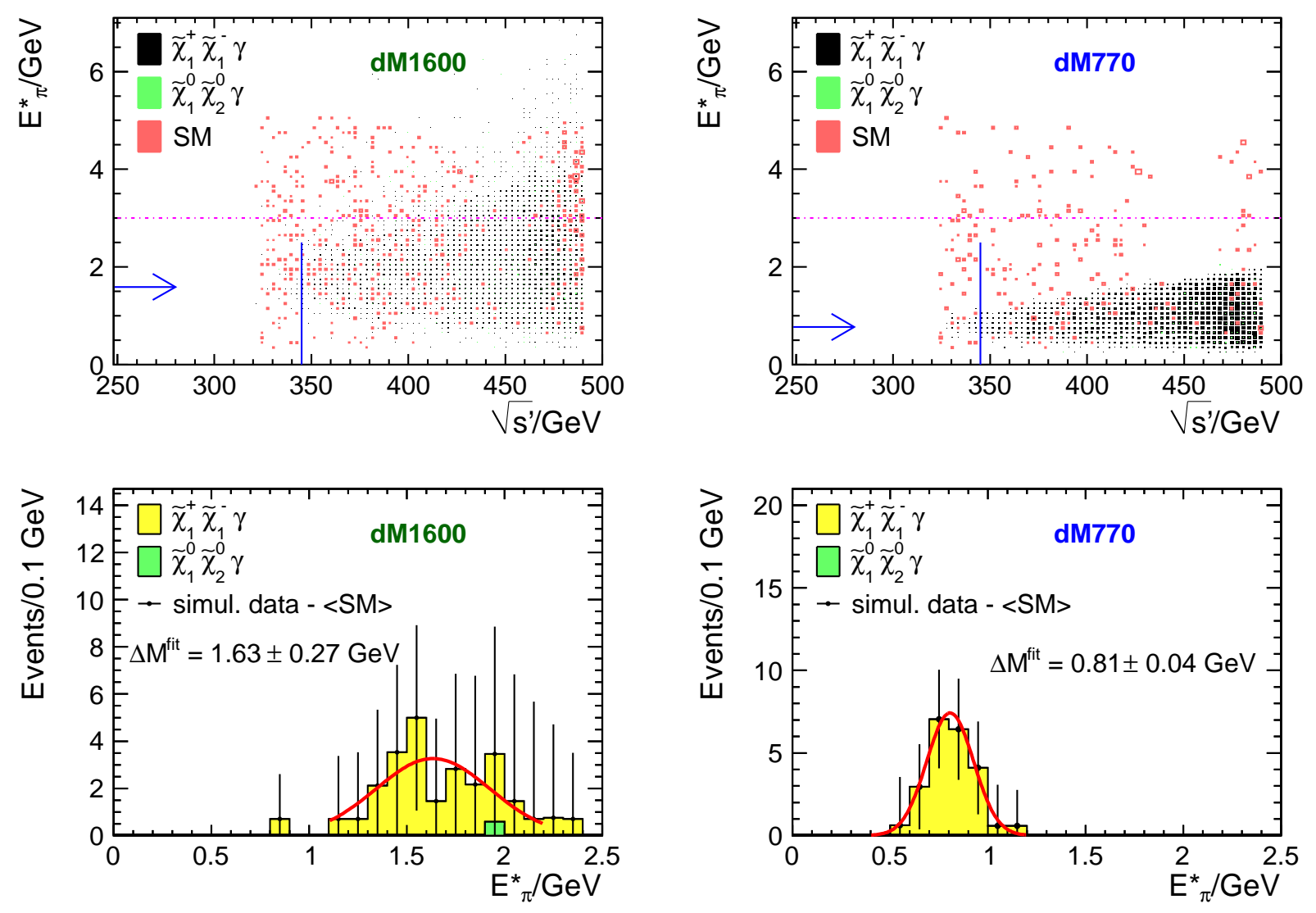

Figure 8: Measurement of the chargino-LSP mass difference for an integrated luminosity of $500 \mathrm{fb}^{-1}$ with $P\left(e^{+}, e^{-}\right)=(+30 \%,-80 \%)$. The upper row shows $E_{\pi}^{*}$ vs. $\sqrt{s^{\prime}}$. The horizontal dashed line indicates the cut on $E_{\pi}^{*}$, the vertical line the cut on $\sqrt{s^{\prime}}$. The arrow indicates the input value of the chargino-LSP mass difference. The lower row the $E_{\pi}^{*}$ after cutting on $\sqrt{s^{\prime}}$. Left: dM1600 scenario; Right: dM770 scenario.

The mass difference is reconstructed as

$$
\begin{array}{rlll}
\mathrm{dM} 1600 & : & \Delta M_{\widetilde{\chi}_{1}^{ \pm}-\widetilde{\chi}_{1}^{0}}^{\mathrm{rec}}=1630 \pm 270 \mathrm{MeV} & \left(\Delta M_{\widetilde{\chi}_{1}^{ \pm}-\widetilde{\chi}_{1}^{0}}^{\text {true }}=1600 \mathrm{MeV}\right), \\
\mathrm{dM} 770 & : \Delta M_{\widetilde{\chi}_{1}^{ \pm}-\widetilde{\chi}_{1}^{0}}^{\text {rec }}=810 \pm 40 \mathrm{MeV} & \left(\Delta M_{\widetilde{\chi}_{1}^{ \pm}-\widetilde{\chi}_{1}^{0}}^{\text {true }}=770 \mathrm{MeV}\right) .
\end{array}
$$

These values agree with the input mass differences within the uncertainty.

\section{Measurement of the polarised chargino cross sections}

The achievable precision on the polarised cross sections is estimated based on the event counts in the final row of Table 5 , i.e. after requiring $E_{\text {miss }}>350 \mathrm{GeV}$, according to

$$
\frac{\delta \sigma}{\sigma}=\frac{1}{\sqrt{\epsilon \cdot \pi \cdot \sigma \cdot \int \mathcal{L} d t}} .
$$

Table 6 displays the corresponding efficiencies $(\epsilon)$ and purities $(\pi)$ as well as the resulting precision on the cross sections assuming an integrated luminosity of $500 \mathrm{fb}^{-1}$ for each polarisation configuration. Since the efficiencies in this case refer to the total number of produced chargino events regardless of their decay channel, 


\begin{tabular}{lcccc}
\hline & \multicolumn{2}{c}{$P\left(e^{+}, e^{-}\right)=(+30 \%,-80 \%)$} & $P\left(e^{+}, e^{-}\right)=(-30 \%,+80 \%)$ \\
\hline & $\mathrm{dM} 1600$ & $\mathrm{dM} 770$ & $\mathrm{dM} 1600$ & $\mathrm{dM} 770$ \\
\hline $\mathcal{L} \mathcal{L} d t$ & $500 \mathrm{fb}^{-1}$ & $500 \mathrm{fb}^{-1}$ & $500 \mathrm{fb}^{-1}$ & $500 \mathrm{fb}^{-1}$ \\
$\sigma$ & $78.7 \mathrm{fb}$ & $77.0 \mathrm{fb}$ & $20.4 \mathrm{fb}$ & $19.9 \mathrm{fb}$ \\
BR of selected mode $(\mathrm{s})$ & $30.5 \%$ & $34.7 \%$ & $30.5 \%$ & $34.7 \%$ \\
\hline efficiency $\epsilon$ & $9.9 \%$ & $12.1 \%$ & $9.5 \%$ & $12.2 \%$ \\
purity $\pi$ & $70.1 \%$ & $85.3 \%$ & $36.4 \%$ & $56.1 \%$ \\
\hline$\delta \sigma / \sigma$ & $1.9 \%$ & $1.6 \%$ & $5.3 \%$ & $3.8 \%$ \\
\hline
\end{tabular}

Table 6: Efficiency, purity, and relative statistical precision on the visible cross section for charginos. The cross section values given here are higher than those displayed in Fig. 2 and 3 since in the event generation step no generator-level cut on the energy of the ISR photon was applied, cf. Sec. 3.2

we display for information the branching ratios of the decay modes to which the selections are tailored, as well as the assumed production cross sections.

For the signal-enriching choice of $P\left(e^{+}, e^{-}\right)=(+30 \%,-80 \%)$, the cross section can be determined to $1.9 \%(1.6 \%)$ in the dM1600 (dM770) scenario. For $P\left(e^{+}, e^{-}\right)=(-30 \%,+80 \%)$, the signal cross sections are lower, while the dominating SM background processes do not depend on the polarisation. Therefore the resolution worsens to $5.3 \%$ and $3.8 \%$ in the two scenarios, respectively. These statistical uncertainties refer to the cross section for the selected semi-leptonic final state. The branching ratio is determined completely by the mass difference. Therefore the total production cross section can be extracted, where the additional uncertainty on the branching ratio from the limited knowledge of the mass difference needs to be included in addition. The corresponding numbers will be given in Section 5

\subsection{Neutralino selection}

The neutralino events are selected by the decay to photon and the LSP. For the dM770 scenario, this is the dominant decay by far, occurring in $74 \%$ of the events. In the dM1600 case, hadronic decay modes dominate, but the branching ratio for the very clean radiative decay is still sizeable with $24 \%$. Therefore, after the common preselection described in Section 4.1. events with only photons in the final state are selected by applying a veto against reconstructed leptons and hadrons. All "soft" photons, i.e. all photons apart from the hard ISR photon candidate, are required to fulfil $\left|\cos \theta_{\gamma_{\text {soft }}}\right|<0.85$. If more than one "soft" photon is reconstructed in addition to the hard ISR photon, then the candidate with the highest transverse momentum is considered as the neutralino decay photon. In analogy to $E_{\pi}^{*}$ in the chargino case, the variable $E_{\gamma_{\text {soft }}}^{*}$ is defined as

$$
E_{\gamma_{\text {soft }}}^{*}=\frac{\left(\sqrt{s}-E_{\gamma}\right) E_{\gamma_{\text {soft }}}+\vec{p}_{\gamma_{\text {soft }}} \cdot \vec{p}_{\gamma}}{\sqrt{s^{\prime}}},
$$

and $E_{\gamma_{\text {soft }}}^{*}>0.5 \mathrm{GeV}$ is required at the final selection step. The resulting event counts are summarised in Table 7 .

\section{Neutralino mass reconstruction}

As in the chargino case, the mass of the $\widetilde{\chi}_{2}^{0}$ is reconstructed from $\sqrt{s^{\prime}}$ determined using the ISR photon. As opposed to the chargino case, the masses of the two produced neutralinos are not equal, but their difference is smaller than the resolution of the $\sqrt{s^{\prime}}$ method. Therefore we use the approximation of two equal mass particles being produced and calibrate the method in the end.

Figure 9 shows the $\sqrt{s^{\prime}}$ distribution obtained in both scenarios. The signal is clearly visible above the background, which has only a negligible contribution from chargino production. Like in the chargino case, 


\begin{tabular}{lccccccc}
\hline & \multicolumn{2}{c}{$\mathrm{dM} 1600$} & \multicolumn{2}{c}{$\mathrm{dM} 770$} & \multicolumn{3}{c}{ Standard Model } \\
\hline & $\tilde{\chi}_{1}^{+} \widetilde{\chi}_{1}^{-} \gamma$ & $\widetilde{\chi}_{1}^{0} \widetilde{\chi}_{2}^{0} \gamma$ & $\tilde{\chi}_{1}^{+} \widetilde{\chi}_{1}^{-} \gamma$ & $\widetilde{\chi}_{1}^{0} \widetilde{\chi}_{2}^{0} \gamma$ & $e e \rightarrow 2,4,6 \mathrm{f}$ & $e \gamma \rightarrow 3,5 \mathrm{f}$ & $\gamma \gamma \rightarrow 2,4 \mathrm{f}$ \\
\hline after preselection & 19872 & 6365 & 21558 & 6872 & 5731 & $1.1837 \times 10^{5}$ & $3.3051 \times 10^{5}$ \\
Photon final state & 53 & 1733 & 155 & 5224 & 399 & 1217 & 2254 \\
$\mid \cos \theta_{\gamma_{\text {soft }} \mid<0.85}$ & 38 & 1467 & 120 & 4538 & 233 & 800 & 1145 \\
$E_{\gamma_{\text {soft }}}>0.5 \mathrm{GeV}$ & 19 & 1395 & 22 & 4095 & 109 & 242 & 413 \\
\hline$E_{\text {miss }}>350 \mathrm{GeV}$ & 19 & 1395 & 22 & 4095 & 90 & 180 & 384 \\
\hline
\end{tabular}

Table 7: Number of events passing the final neutralino selection, following the preselection in Tab. 4 for an integrated luminosity of $500 \mathrm{fb}^{-1}$ and $P\left(e^{+}, e^{-}\right)=(+30 \%,-80 \%)$.
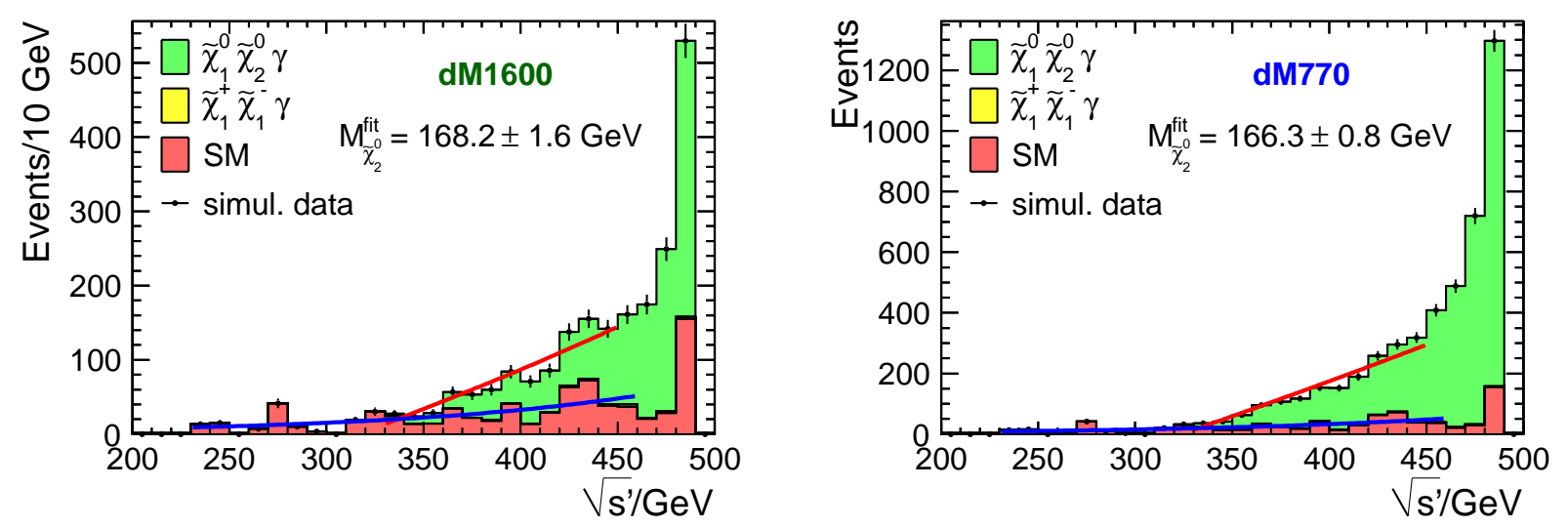

Figure 9: Distribution of the reduced centre-of-mass energy $\left(\sqrt{s^{\prime}}\right)$ of the system recoiling against the hard ISR photon for all events passing the neutralino selection for an integrated luminosity of $500 \mathrm{fb}^{-1} \mathrm{with}$ $P\left(e^{+}, e^{-}\right)=(+30 \%,-80 \%) \cdot M_{\widetilde{\chi}_{2}^{0}}$ is determined from fitting the sum (red curve) of a straight line for the signal and the background parametrisation (blue curve) to the distribution near the endpoint. Left: dM1600 scenario; Right: dM770 scenario.

the SM background is fitted first with an exponential (blue line). In a second step, a straight line is added on top of the background to model the signal contribution and fitted to the simulated data in the endpoint region (red line). Again the parameters of the SM-background function are fixed to the values obtained from the SM-only fit in the wider $\sqrt{s^{\prime}}$ window. The neutralino mass is fitted to $168.2 \pm 1.6 \mathrm{GeV}$ in the dM1600 scenario and to $166.3 \pm 0.8 \mathrm{GeV}$ in the dM770 case. Both numbers agree with the input values within $1-1.5 \sigma$.

Again the correlation between fitted mass and input mass is investigated, resulting in the calibration curve displayed in Figure 10, Since in both scenarios the same decay modes are selected, there is no reason to assume a different calibration behaviour in the other scenario. Therefore the same calibration curve is used. Since its slope is only about 0.5 , the final statistical uncertainty on the calibrated neutralino masses grows by a factor of 2 . After applying the calibration, the final results are

$$
\begin{array}{rlll}
\mathrm{dM} 1600 & : & M_{\widetilde{\chi}_{2}^{0}}^{\text {cal }}=169.6 \pm 3.3 \mathrm{GeV} & \left(M_{\widetilde{\chi}_{2}^{0}}^{\text {true }}=166.9 \mathrm{GeV}\right) \\
\mathrm{dM} 770 & : \quad M_{\widetilde{\chi}_{2}^{0}}^{\text {cal }}=165.7 \pm 1.6 \mathrm{GeV} & \left(M_{\widetilde{\chi}_{2}^{0}}^{\text {true }}=167.6 \mathrm{GeV}\right)
\end{array}
$$

\section{Measurement of the polarised neutralino cross sections}

The achievable precision on the polarised cross sections is estimated like in the chargino case, based on the event counts given in Table 7 . Table 8 displays the corresponding efficiencies and purities as well as the 


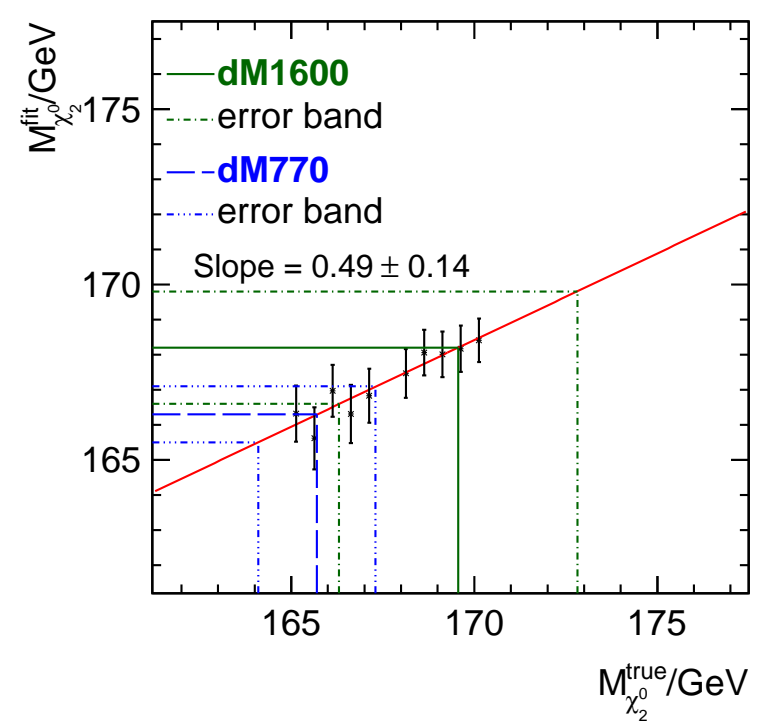

$$
\begin{array}{cc}
\text { dM1600 } & \text { dM770 } \\
M_{\tilde{\chi}_{2}^{0}}^{\text {true }}=166.9 \mathrm{GeV} & \mathrm{M}_{\tilde{\chi}_{2}^{0}}^{\text {true }}=167.6 \mathrm{GeV} \\
M_{\tilde{\chi}_{2}^{0}}^{\text {fit }}=168.2 \pm 1.6 \mathrm{GeV} & \mathrm{M}_{\tilde{\chi}_{2}^{0}}^{\text {fit }}=166.3 \pm 0.8 \mathrm{GeV} \\
M_{\tilde{\chi}_{2}^{0}}^{\text {cal }}=169.6 \pm 3.3 \mathrm{GeV} & \mathrm{M}_{\tilde{\chi}_{2}^{0}}^{\text {cal }}=165.7 \pm 1.6 \mathrm{GeV}
\end{array}
$$

Figure 10: Calibration of the $\sqrt{s^{\prime}}$ method for the neutralino mass determination. Since here the same decay mode is selected for both scenarios, the same calibration curve can be used.

resulting precision on the cross sections assuming an integrated luminosity of $500 \mathrm{fb}^{-1}$ for each polarisation configuration. Since the efficiencies in this case refer to the total number of produced neutralino events regardless of their decay channel, the branching ratios of the radiative decay are displayed in addition for information, as well as the assumed production cross sections.

\begin{tabular}{lcccc}
\hline & \multicolumn{2}{c}{$P\left(e^{+}, e^{-}\right)=(+30 \%,-80 \%)$} & \multicolumn{2}{c}{$P\left(e^{+}, e^{-}\right)=(-30 \%,+80 \%)$} \\
\hline & $\mathrm{dM} 1600$ & $\mathrm{dM} 770$ & $\mathrm{dM} 1600$ & $\mathrm{dM} 770$ \\
\hline $\int \mathcal{L} d t$ & $500 \mathrm{fb}^{-1}$ & $500 \mathrm{fb}^{-1}$ & $500 \mathrm{fb}^{-1}$ & $500 \mathrm{fb}^{-1}$ \\
$\sigma$ & $49.0 \mathrm{fb}$ & $48.4 \mathrm{fb}$ & $38.9 \mathrm{fb}$ & $38.4 \mathrm{fb}$ \\
BR of selected mode & $23.6 \%$ & $74.0 \%$ & $23.6 \%$ & $74.0 \%$ \\
\hline efficiency $\epsilon$ & $5.8 \%$ & $17.1 \%$ & $6.0 \%$ & $17.2 \%$ \\
purity $\pi$ & $67.4 \%$ & $85.8 \%$ & $62.3 \%$ & $82.5 \%$ \\
\hline$\delta \sigma / \sigma$ & $3.2 \%$ & $1.7 \%$ & $3.7 \%$ & $1.9 \%$ \\
\hline
\end{tabular}

Table 8: Efficiency, purity, and relative statistical precision on the visible cross section for associated neutralino production. The cross section values given here are higher than those displayed in Fig. 2 and 3 since in the event generation step no generator-level cut on the energy of the ISR photon was applied, cf. Sec. 3.2

For neutralino production, the total cross section is much less sensitive to beam polarisation than for chargino production, cf. Figure 2, Therefore, the precision depends only marginally on the beam polarisation. Due to the different branching ratios for the radiative decay, the cross sections can be determined more precisely, to about $1.7 \%$ in the dM770 scenario, while the measurements are about a factor two less precise in the dM1600 case. 


\begin{tabular}{lrr}
\hline Observable & $\mathrm{dM} 1600$ & $\mathrm{dM} 770$ \\
\hline$M_{\widetilde{\chi}_{1}^{ \pm}}[\mathrm{GeV}]$ & $166.2 \pm 2.0$ & $167.3 \pm 1.5$ \\
$M_{\widetilde{\chi}_{2}^{0}}[\mathrm{GeV}]$ & $169.6 \pm 3.3$ & $165.7 \pm 1.6$ \\
$M_{\widetilde{\chi}_{1}^{ \pm}}-M_{\widetilde{\chi}_{1}^{0}}[\mathrm{GeV}]$ & $1.63 \pm 0.27$ & $0.81 \pm 0.04$ \\
$\delta \sigma / \sigma\left(\widetilde{\chi}_{1}^{+} \widetilde{\chi}_{1}^{-} \gamma\right)_{(-0.3,0.8)}^{500}$ & $16 \%$ & $4.3 \%$ \\
$\delta \sigma / \sigma\left(\widetilde{\chi}_{1}^{+} \widetilde{\chi}_{1}^{-} \gamma\right)_{(0.3,-0.8)}^{500}$ & $15 \%$ & $2.6 \%$ \\
$\delta \sigma / \sigma\left(\widetilde{\chi}_{1}^{+} \widetilde{\chi}_{1}^{-} \gamma\right)_{(-0.3,0.8)}^{350}$ & $33 \%$ & $20 \%$ \\
$\delta \sigma / \sigma\left(\widetilde{\chi}_{1}^{+} \widetilde{\chi}_{1}^{-} \gamma\right)_{(0.3,-0.8)}^{350}$ & $18 \%$ & $9.0 \%$ \\
$\delta \sigma / \sigma\left(\widetilde{\chi}_{1}^{0} \widetilde{\chi}_{2}^{0} \gamma\right)_{(-0.3,0.8)}^{500}$ & $4.0 \%$ & $2.4 \%$ \\
$\delta \sigma / \sigma\left(\widetilde{\chi}_{1}^{0} \widetilde{\chi}_{2}^{0} \gamma\right)_{(0.3,-0.8)}^{500}$ & $3.5 \%$ & $2.3 \%$ \\
$\delta \sigma / \sigma\left(\widetilde{\chi}_{1}^{0} \widetilde{\chi}_{2}^{0} \gamma\right)_{(-0.3,0.8)}^{350}$ & $20 \%$ & $10 \%$ \\
$\delta \sigma / \sigma\left(\widetilde{\chi}_{1}^{0} \widetilde{\chi}_{2}^{0} \gamma\right)_{(0.3,-0.8)}^{350}$ & $18 \%$ & $9.4 \%$ \\
\hline
\end{tabular}

Table 9: Observables used in the fit: masses with uncertainties taken from Eqs. (17), (18), (20), (21), (24), (25); relative cross sections uncertainties from Tabs. 6 and 8 including systematic uncertainty due to branching ratios as discussed in text. The subscript on $\delta \sigma / \sigma$ denotes the beam polarisation $P\left(e^{+}, e^{-}\right)$and the superscript the centre-of-mass energy in $\mathrm{GeV}$.

\section{Parameter determination}

In the final step of the analysis, we estimate the accuracy to which the underlying MSSM parameters can be determined. As discussed in Section 2.2 there are four real parameters that define the chargino and neutralino sector at tree level:

$$
M_{1}, \quad M_{2}, \quad \mu, \quad \tan \beta .
$$

We consider both $M_{1}>0$ and $M_{1}<0$, while the sign of $\mu$ cannot be resolved due to its negligible impact on observables. They can be extracted using the measurements discussed in the previous section: the masses of the light chargino $\widetilde{\chi}_{1}^{ \pm}$and the neutralino $\widetilde{\chi}_{2}^{0}$, the mass difference $M_{\widetilde{\chi}_{1}^{ \pm}}-M_{\widetilde{\chi}_{1}^{0}}$, the chargino pair production cross section and the $\widetilde{\chi}_{1}^{0} \widetilde{\chi}_{2}^{0}$ production cross section. The respective values and uncertainties are summarised in Tab. 9. Since we have not performed a detector simulation for $\sqrt{s}=350 \mathrm{GeV}$, the uncertainties in this case are obtained by rescaling the $\sqrt{s}=500 \mathrm{GeV}$ result. The ratio of the cross sections is $\sim 30$ and we conservatively scale the errors by $\sqrt{30}$. One should note that at $\sqrt{s}=350 \mathrm{GeV}$ the $\mathrm{SM}$ background contributions are expected to be smaller, therefore the errors are likely to be lower than our current estimate.

The cross section errors obtained in Sec. 4 did not take into account the uncertainty on the branching ratios. The branching ratios only depend on the available phase space for the decay of the virtual $W$ boson for charginos, given by the chargino-LSP mass difference. With the previously obtained precision on the mass difference, the uncertainty on the BR of the selected decay modes has to be taken into account. In case of the dM1600 scenario, due to the significant uncertainty on the chargino-LSP mass difference, it amounts to $15 \%$ for the chargino cross section measurement. For the dM770 scenario it is $2 \%$, comparable to the experimental uncertainty. These additional uncertainties are included in Table 9

Using Minuit [58] we minimise a $\chi^{2}$ function defined as

$$
\chi^{2}=\sum_{i}\left|\frac{\mathcal{O}_{i}-\overline{\mathcal{O}}_{i}}{\delta \mathcal{O}_{i}}\right|^{2}
$$

where the sum runs over the input observables $\mathcal{O}_{i}$, with their corresponding experimental uncertainties $\delta \mathcal{O}_{i}$. The theoretical values calculated using the fitted MSSM parameters, Eq. (26), are denoted by $\overline{\mathcal{O}}_{i}$. Note 


\begin{tabular}{|c|c|c|c|c|c|c|}
\hline & & & \multicolumn{2}{|c|}{$\sqrt{s}=500 \mathrm{GeV}$} & \multicolumn{2}{|c|}{$\sqrt{s}=350 \& 500 \mathrm{GeV}$} \\
\hline & & input & lower & upper & lower & upper \\
\hline \multirow{3}{*}{ 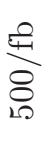 } & $\left|M_{1}\right|[\mathrm{TeV}]$ & 1.7 & $\sim 0.8(0.4)$ & no & $\sim 0.8(0.4)$ & no \\
\hline & $M_{2}[\mathrm{TeV}]$ & 4.36 & $\sim 1.5(1.0)$ & no & $\sim 1.5(1.0)$ & no \\
\hline & $\mu[\mathrm{GeV}]$ & 165.66 & 165.2 & 172.5 & 165.4 & 170.2 \\
\hline \multirow{3}{*}{$\frac{\tilde{\sigma}}{\sim}$} & $\left|M_{1}\right|[\mathrm{TeV}]$ & 1.7 & $\sim 1.0(0.4)$ & $\sim 6.0(0.6)$ & $\sim 1.0(0.4)$ & $\sim 6.0(0.6)$ \\
\hline & $M_{2}[\mathrm{TeV}]$ & 4.36 & $\sim 2.5(3.5)$ & $\sim 8.5(\mathrm{no})$ & $\sim 2.5(3.5)$ & $\sim 8.5(\mathrm{no})$ \\
\hline & $\mu[\mathrm{GeV}]$ & 165.66 & 166.2 & 170.1 & 166.4 & 170.0 \\
\hline
\end{tabular}

Table 10: The 1- $\sigma$ allowed ranges for the parameter fit in scenario dM1600. $\tan \beta$ is allowed to vary in the range $[1,60]$. Values in parentheses are for $M_{1}<0$. Determination of the parameters for integrated luminosities of $\int \mathcal{L} d t=500 \mathrm{fb}^{-1}$ and $2 \mathrm{ab}^{-1}$ per polarisation configuration is shown. The high-luminosity fit also includes the mass difference between neutralinos, $M_{\widetilde{\chi}_{2}^{0}}-M_{\widetilde{\chi}_{1}^{0}}$. For an exact shape of the allowed $M_{1}-M_{2}$ region, see Fig. 11. The input values, see Eq. (12), are also shown.

that throughout the simulation the one-loop corrected masses, given by Eqs. (12) and (13), were used. The difference between the tree-level values and the ones obtained from SOFTSUSY is accordingly taken into account in the fit in order to ensure consistency. The variation of the observables, with respect to the central values, on the SUSY parameters, Eq. (26), is in the following calculated at the tree level. Therefore, the results of the fit are to be understood as the tree-level fit.

It turns out that using the experimental information one cannot constrain $\tan \beta$. This is not very surprising, as with the values of parameters used here, there is very little mixing between higgsinos and gauginos which could have provided some sensitivity to $\tan \beta$. As can be seen from Eq. (7), the chargino and neutralino masses depend only on $\sin 2 \beta$ and the sensitivity is rather weak, especially in the large $\tan \beta$ limit. In order to have a converging fit we therefore fix $\tan \beta$ to values in the range $\tan \beta \in[1,60]$ and fit the remaining three parameters of Eq. (26). In principle, the cross sections depend also on the sneutrino and selectron masses but since the couplings to higgsinos are negligible, no dependence on these parameters enters our observables.

The results of the fit are shown in Tables 10 and 11. The crucial observable for the determination of $M_{1}$ and $M_{2}$ is the mass difference between the LSP and the light chargino. On the other hand, the cross section measurements improve the determination of the $\mu$ parameter. In general, only lower limits can be obtained for $\left|M_{1}\right|$ and $M_{2}$, which are given in Tables 10 and 11 for positive and negative $M_{1}$. However, it turns out that $M_{1}$ and $M_{2}$ are strongly correlated and the allowed regions are only narrow strips in the $M_{1}-M_{2}$ parameter space, see Figs. 11 and 12. The $\mu$ parameter can be determined with an accuracy $\sim 2.5 \mathrm{GeV}$ in dM770 scenario and $6.8 \mathrm{GeV}$ in dM1600 scenario.

Inclusion of the cross section measurements at $\sqrt{s}=350 \mathrm{GeV}$ only affects the determination of the $\mu$ parameter. The low cross section results in a large error, larger by a factor of 5.5 than for $\sqrt{s}=500 \mathrm{GeV}$. However, being close to the production threshold and on the quickly rising slope of the cross section, see Fig. 2. helps to improve the determination of the $\mu$ parameter in both scenarios. Taking the cross section at a higher centre-of-mass energy, e.g. $\sqrt{s}=370 \mathrm{GeV}$, does not yield a significant improvement. The increased statistics does not compensate the advantage of being close to the production threshold. The masses and mass differences require larger statistics, and therefore, measurements at higher centre-of-mass energies (e.g. at $500 \mathrm{GeV}$ ) are indispensable. Finally, we note that the main driver for the rather high $\mu$ error is the variation of $\tan \beta$, which shifts the central values of the $\mu$ parameter by approximately $\sim 1 \mathrm{GeV}$. This additional dependence will remain regardless of additional measurements and/or increased accuracy.

In order to better understand the interdependence of the $M_{1}$ and $M_{2}$ determination, in the top panels of Figs. 11 and 12 we show the $1-\sigma$ contours in the $M_{1}-M_{2}$ plane for an integrated luminosity of $500 \mathrm{fb}^{-1}$. Different contours correspond to different values of $\tan \beta$. For low values of either $M_{1}$ or $M_{2}$ the allowed region for the other parameter extends to arbitrary high values and cannot be reliably constrained. Significant 


\begin{tabular}{|c|c|c|c|c|c|c|}
\hline & & & \multicolumn{2}{|c|}{$\sqrt{s}=500 \mathrm{GeV}$} & \multicolumn{2}{|c|}{$\sqrt{s}=350 \& 500 \mathrm{GeV}$} \\
\hline & & input & lower & upper & lower & upper \\
\hline \multirow{3}{*}{$\frac{c}{8}$} & $\left|M_{1}\right|[\mathrm{TeV}]$ & 5.3 & $\sim 2(0.3)$ & no & $\sim 2(0.3)$ & no \\
\hline & $M_{2}[\mathrm{TeV}]$ & 9.51 & $\sim 3(1.2)$ & no & $\sim 3(1.2)$ & no \\
\hline & $\mu[\mathrm{GeV}]$ & 167.22 & 164.8 & 167.8 & 165.2 & 167.7 \\
\hline \multirow{3}{*}{$\frac{\widetilde{\sigma}}{\text { N }}$} & $\left|M_{1}\right|[\mathrm{TeV}]$ & 5.3 & $\sim 3$ & no & $\sim 3$ & no \\
\hline & $M_{2}[\mathrm{TeV}]$ & 9.51 & $\sim 7$ & $\sim 15$ & $\sim 7$ & $\sim 15$ \\
\hline & $\mu[\mathrm{GeV}]$ & 167.22 & 165.2 & 167.4 & 165.3 & 167.4 \\
\hline
\end{tabular}

Table 11: The 1- $\sigma$ allowed ranges for the parameter fit in scenario dM770. $\tan \beta$ is allowed to vary in the range $[1,60]$. Values in parentheses are for $M_{1}<0$ if solutions exist. Determination of the parameters for integrated luminosities of $\int \mathcal{L} d t=500 \mathrm{fb}^{-1}$ and $2 \mathrm{ab}^{-1}$ per polarisation configuration is shown. The high-luminosity fit also includes the mass difference between neutralinos, $M_{\widetilde{\chi}_{2}^{0}}-M_{\widetilde{\chi}_{1}^{0}}$. For an exact shape of the allowed $M_{1}-M_{2}$ region, see Fig. 12. The input values, see Eq. (13), are also shown.

variation with $\tan \beta$ is also visible, especially in case of scenario dM770. Both cases of the sign of the $M_{1}$ parameter are considered. In general, for $M_{1}<0$ lower absolute values of gaugino masses are consistent with data. In the most extreme cases, when $M_{1} \gtrsim-800 \mathrm{GeV}$, a direct production of $\widetilde{\chi}_{3}^{0}$ could be possible at the ILC with an increased centre-of-mass energy. We also note that for negative $M_{1}$ low values of $\tan \beta$ are experimentally excluded since they lead to an inverted mass hierarchy between the light chargino and the lightest neutralino, $m_{\tilde{\chi}_{1}^{ \pm}}<m_{\tilde{\chi}_{1}^{0}}$.

Finally, we assess how much of an improvement can be expected after a high-luminosity run with a total of $\int \mathcal{L} d t=2 \mathrm{ab}^{-1}$ integrated luminosity at $\sqrt{s}=500 \mathrm{GeV}$. We assume that with the increased luminosity the experimental errors would be reduced by a factor of 2 . We furthermore include the measurement of the mass difference $M_{\widetilde{\chi}_{2}^{0}}-M_{\widetilde{\chi}_{1}^{0}}$, which was not studied here. For the $\mu$ parameter the increased luminosity narrows the allowed region by $2-3.5 \mathrm{GeV}$, as can be seen in the last rows of Tabs. 10 and 11 In case of the $M_{1}$ and $M_{2}$ parameters the results are shown in the bottom panels of Figs. 11 and 12 for scenarios dM1600 and dM770, respectively. The neutralino mass difference, $M_{\widetilde{\chi}_{2}^{0}}-M_{\widetilde{\chi}_{1}^{0}}$, helps to break the correlation between $M_{1}$ and $M_{2}$ from the chargino-LSP mass difference, since it exhibits a different dependence on the fundamental parameters, in particular by introducing $\tan \beta$, see Eqs. (9) and (11). Hence, the inclusion of the neutralino mass difference helps to constrain the parameters in the low $\tan \beta$ regime, where the $\tan \beta$ dependence is numerically most significant. This effect would not be achieved by solely increasing the luminosity. The absolute mass measurements, which are obviously improved with higher statistics, have only a very limited impact on determination of the multi-TeV parameters, $M_{1}$ and $M_{2}$. The neutralino mass difference, due to its different dependence on the fundamental parameters, provides important additional information to the fit. This underlines the importance of including this measurement in future analyses.

For the scenario dM1600, $M_{2}$ is now constrained in the $2.5-8.5 \mathrm{TeV}$ range, while $M_{1}>0$ in the $1-6 \mathrm{TeV}$ range. If $M_{1}<0$ its value is confined in a small range, $-700 \mathrm{GeV} \lesssim M_{1} \lesssim-400 \mathrm{GeV}$ for moderate values of $\tan \beta, 8 \leq \tan \beta \leq 16$. Again, such a scenario could in principle be probed with an upgraded centre-of-mass energy. Also for dM770 one obtains a visible improvement, but still with rather broad ranges of allowed values for $M_{1}$ and $M_{2}$; however, we only found valid solutions for $M_{1}>0$. In both cases, the contours for other values of $\tan \beta$ are mostly covered by the high $\tan \beta$ contour and are therefore not shown separately. This result is due to the weak dependence on $\tan \beta$ of the complete neutralino/chargino sector, cf. Section 2.2. Additional measurements, involving e.g. third-generation sfermions, would be needed to obtain meaningful constraints on $\tan \beta$. 

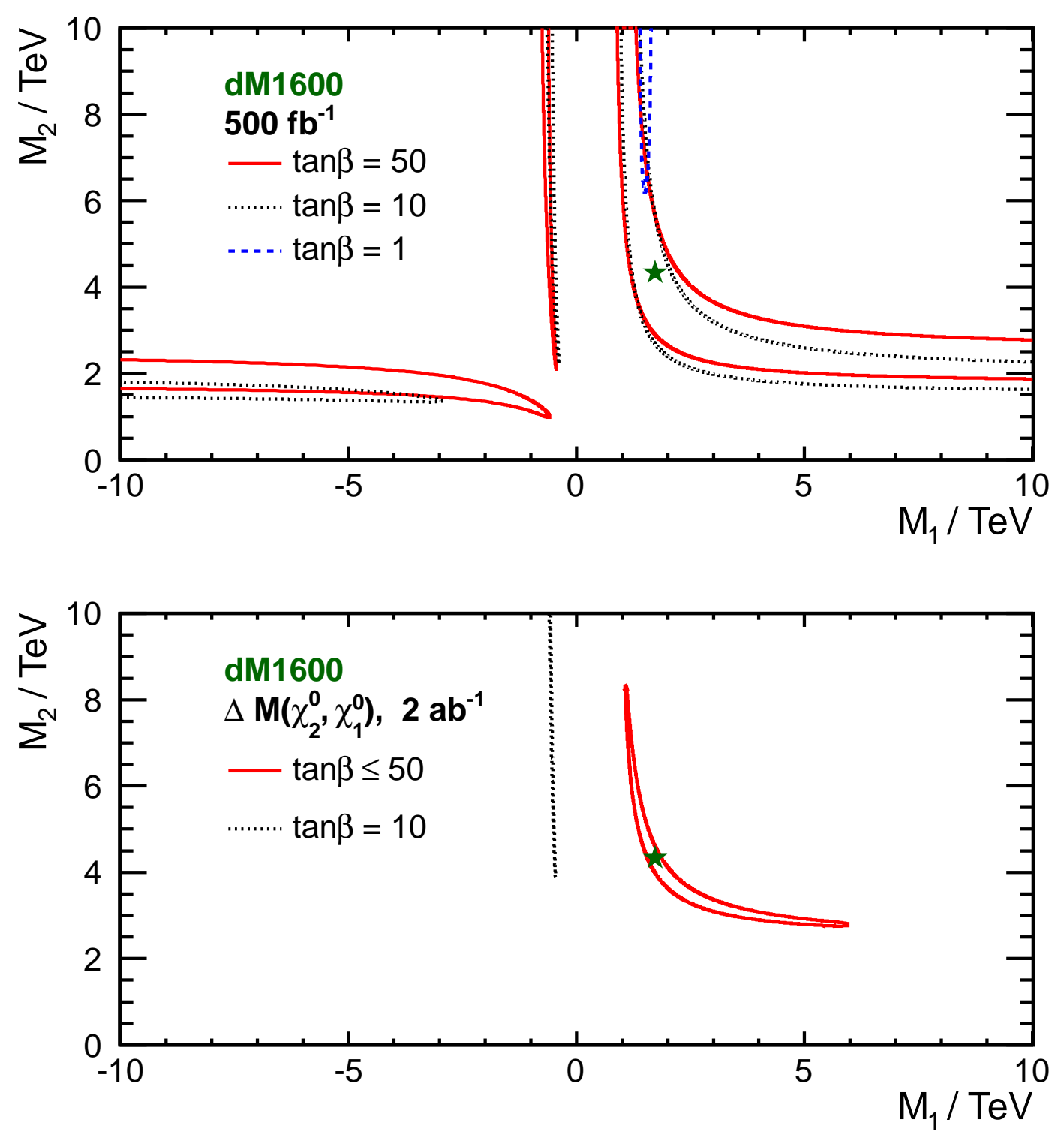

Figure 11: The 1- $\sigma$ contours for determination of $M_{1}$ and $M_{2}$ in scenario dM1600. The input values of $M_{1}$ and $M_{2}$ are indicated by the star, and $(\tan \beta)_{\text {true }}=44$. Top: The fit using input values listed in Tab. 9 for three different values of $\tan \beta$. Bottom: Projected fit results after a high-luminosity run, $\int \mathcal{L} d t=2 \mathrm{ab}^{-1}$, with experimental errors improved by factor 2 and with the mass difference $M_{\widetilde{\chi}_{2}^{0}}-M_{\widetilde{\chi}_{1}^{0}}$ measurement included for a fixed $\tan \beta=50$ and $\tan \beta=10$. For $M_{1}>0$, other values of $\tan \beta$ do not further extend allowed regions and the respective contours are inside the one for $\tan \beta=50$. For $M_{1}<0$, solutions exist only for $8 \leq \tan \beta \leq 16$ near $M_{1} \sim-0.5 \mathrm{TeV}$. 

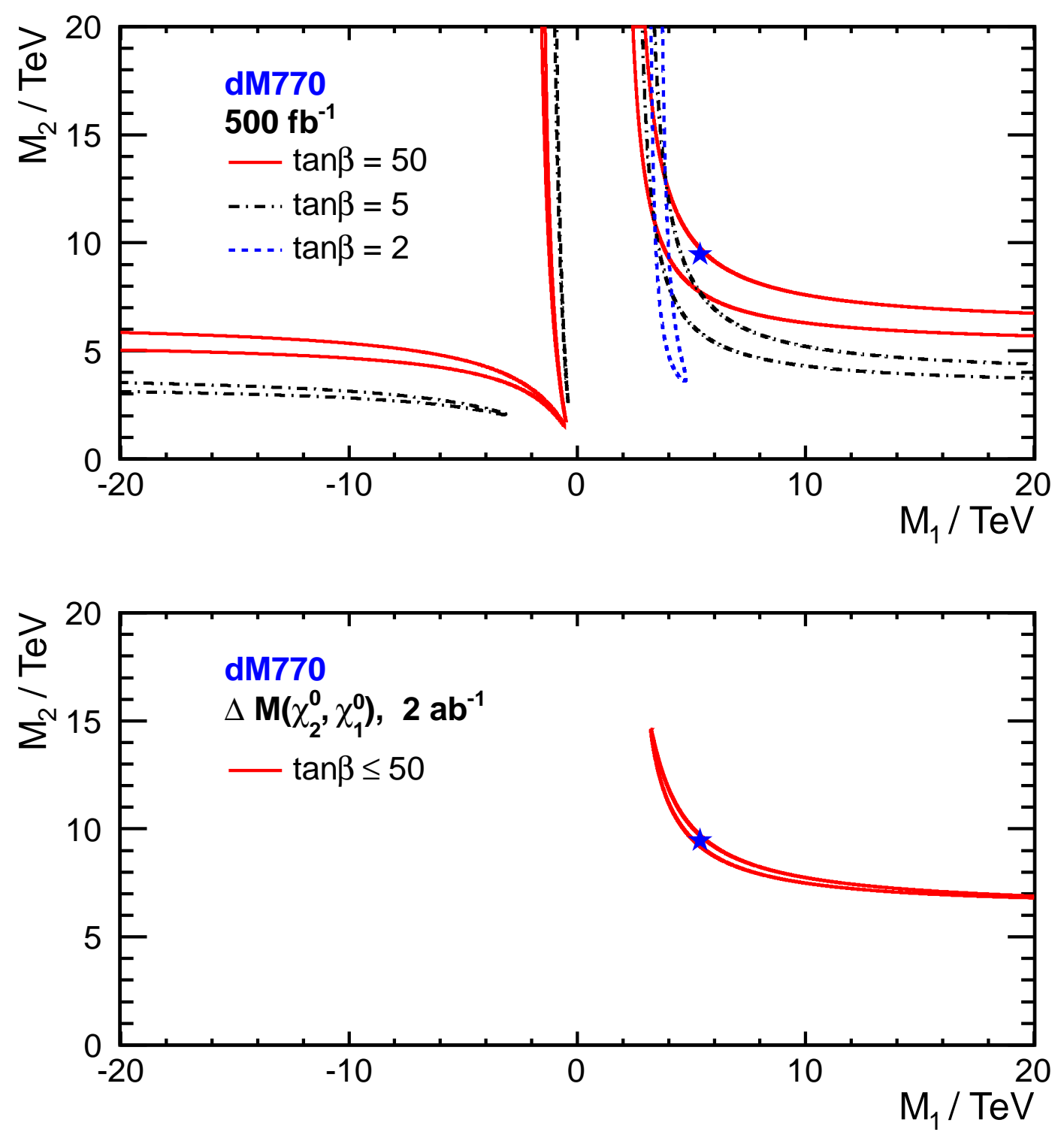

Figure 12: The 1- $\sigma$ contours for determination of $M_{1}$ and $M_{2}$ in scenario dM770. The input values of $M_{1}$ and $M_{2}$ are indicated by the star, and $(\tan \beta)_{\text {true }}=48$. Top: The fit using input values listed in Tab. 9 for three different values of $\tan \beta$. Bottom: Projected fit results after a high-luminosity run, $\int \mathcal{L} d t=2 \mathrm{ab}^{-1}$, with experimental errors improved by factor 2 and with the mass difference $M_{\widetilde{\chi}_{2}^{0}}-M_{\widetilde{\chi}_{1}^{0}}$ measurement included for fixed $\tan \beta=50$. Other values of $\tan \beta$ do not further extend allowed regions and the respective contours are inside the shown one. 


\section{Conclusions}

The current LHC results provide strong bounds on constrained supersymmetric models. In particular, the measured Higgs mass of $m_{h} \approx 126 \mathrm{GeV}$ results in a tension with the naturalness argument. However, in a specific class of scenarios, a rather light $\mu$-parameter in conjunction with a heavy coloured SUSY and gaugino spectrum can be well-motivated from a model-building perspective. Such scenarios result in a strong mass degeneracy between the higgsino-like light neutralinos and chargino, $\widetilde{\chi}_{1,2}^{0}$ and $\widetilde{\chi}_{1}^{ \pm}$. Due to the close mass degeneracies, three-body decays as well as radiative decays are dominant leading to extremely challenging experimental signatures.

We therefore performed a simulation for the ILD detector in two different benchmark scenarios at a centre-of-mass energy of $500 \mathrm{GeV}$, assuming an integrated luminosity of $500 \mathrm{fb}^{-1}$ each for the two relevant polarisation configurations, which corresponds to about 4 years of ILC operation with design accelerator parameters. In both scenarios, the $\mu$-parameter is around $160 \mathrm{GeV}$, but the SUSY gaugino parameters $M_{1}$ and $M_{2}$ are in the multi-TeV region. This leads to mass differences between the light chargino and lightest neutralino of about $1.6 \mathrm{GeV}$ and $770 \mathrm{MeV}$ in the two benchmarks, respectively. These small mass differences lead to a very soft transverse momentum spectrum of the visible decay products, which ends at 5 and $2 \mathrm{GeV}$ for the two different scenarios. In this kinematic regime, background processes from collisions involving the photon component of the electron and positron beams are about 5 orders of magnitude larger than the signal. However, they can be heavily reduced by requiring the signal to be accompanied by a hard ISR photon.

In conjunction with the known beam energy, the $\widetilde{\chi}_{1}^{ \pm}$and $\widetilde{\chi}_{2}^{0}$ masses can be determined with a precision between 1-2\% from the reduced centre-of-mass energy of the system recoiling against the photon. The mass difference with the LSP can be reconstructed by boosting the visible decay products into the rest frame of the produced gaugino pair. This allows one to clearly resolve the lightest and next-to-lightest SUSY particles and to determine the corresponding mass difference in the two benchmarks with a relative accuracy of $17 \%$ and $5 \%$, respectively. Finally, the polarised cross sections can be measured with an accuracy of a few percent. The polarisation dependency, in particular the scaling factor between polarised and unpolarised cross sections, gives a clear indication of the higgsino nature.

In the last step of this analysis, we investigated whether the relevant SUSY parameters, namely the gaugino mass parameters $M_{1}$ and $M_{2}$, the $\mu$-parameter and $\tan \beta$ can be extracted from the experimental observables. We find that the $\mu$-parameter can be determined to $\pm 4 \%$. In case of $M_{1}$ and $M_{2}$, lower bounds in the multi-TeV range can be set, which depend slightly on the value of $\tan \beta$, which cannot be fixed from these measurements alone. Additional solutions arise if one allows the bino mass parameter to be negative, $M_{1}<0$. Some of these, leading to a light $\widetilde{\chi}_{3}^{0}$, could be probed by direct production of the additional light neutralino. If the measurement uncertainties could be reduced by a factor of 2 , e.g. by increasing the luminosity, or by improving the analysis, and if a measurement of the mass difference between $\widetilde{\chi}_{2}^{0}$ and $\widetilde{\chi}_{1}^{0}$ could be performed with the same precision as for the chargino mass difference, the constraints on $M_{1}$ and $M_{2}$ would be significantly more restrictive and become less dependent on $\tan \beta$.

Our results demonstrate the strong physics potential of the ILC. High precision is mandatory in order to resolve such challenging scenarios that could be easily missed at the LHC. The high experimental precision of thr ILC of course has to be balanced by suitable higher order predictions implemented in a Monte Carlo generator on a fully differential level. First steps in this direction have already been presented in [43, 44], but a full study including decays would be needed and is in the line of future work.

\section{Acknowledgements}

We thank Frank Gäde for providing the tracking efficiency histograms in presence of pair background. We thank the ILC Generator Group for providing the SM Whizard samples. We thank Aoife Bharucha for discussions on loop corrections. We thankfully acknowledge the support by the DFG through the SFB 676 "Particles, Strings and the Early Universe". This work has been partially supported by the MICINN, Spain, under contract FPA2010-17747; Consolider-Ingenio CPAN CSD2007-00042. We thank as well the Comunidad de Madrid through Proyecto HEPHACOS S2009/ESP-1473 and the European Commission under contract PITN-GA-2009-237920. 


\section{References}

[1] G. Aad et al. [ATLAS Collaboration], Phys. Lett. B 716 (2012) 1 arXiv:1207.7214 [hep-ex]].

[2] S. Chatrchyan et al. [CMS Collaboration], Phys. Lett. B 716 (2012) 30 arXiv:1207.7235 [hep-ex]].

[3] G. Aad et al. [ATLAS Collaboration], Eur. Phys. J. C 73 (2013) 2362 arXiv:1212.6149 [hep-ex]]; ATLAS-CONF-2013-054; ATLAS-CONF-2013-047; ATLAS-CONF-2013-037; ATLAS-CONF-2013-026; ATLAS-CONF-2013-024; ATLAS-CONF2013-007; ATLAS-CONF-2012-144; ATLAS-CONF-2012-140.

[4] S. Chatrchyan et al. [CMS Collaboration], Phys. Rev. D 87 (2013) 072001 arXiv:1301.0916 [hep-ex]]; Eur. Phys. J. C 73 (2013) 2404 arXiv:1212.6428 [hep-ex]]; JHEP 1303 (2013) 037 arXiv:1212.6194 [hep-ex]], JHEP 1303 (2013) 111 arXiv:1211.4784 [hep-ex]]; Phys. Lett. B 725 (2013) 243 arXiv:1305.2390 [hep-ex]]; Eur. Phys. J. C 73 (2013) 2568 arXiv:1303.2985 [hep-ex]]; Eur. Phys. J. C 73 (2013) 2493 arXiv:1301.3792 [hep-ex]]; arXiv:1301.2175 [hep-ex]; Phys. Rev. Lett. 111 (2013) 081802 arXiv:1212.6961 [hep-ex]]; CMS-PAS-SUS-13-011; CMS-PAS-SUS-13-008; CMS-PAS-SUS13-007.

[5] [ATLAS Collaboration] ATL-PHYS-PUB-2013-007.

[6] [CMS Collaboration] CMS-PAS-FTR-13-014.

[7] [ATLAS Collaboration], ATLAS-CONF-2013-028, ATLAS-CONF-2013-035 ATLAS-CONF-2013-049 and ATLAS-CONF2013-093.

[8] [CMS Collaboration] PAS-SUS-13-006, PAS-SUS-13-002 and PAS-SUS-13-017.

[9] J. Beringer et al. [Particle Data Group Collaboration], Phys. Rev. D 86 (2012) 010001; A. Heister et al. [ALEPH Collaboration], Phys. Lett. B 533 (2002) 223 hep-ex/0203020]; J. Abdallah et al. [DELPHI Collaboration], Eur. Phys. J. C 31 (2003) 421 hep-ex/0311019]; M. Acciarri et al. [L3 Collaboration], Phys. Lett. B 482 (2000) 31 hep-ex/0002043]; G. Abbiendi et al. [OPAL Collaboration], Eur. Phys. J. C 35 (2004) 1 hep-ex/0401026].

[10] M. Papucci, J. T. Ruderman and A. Weiler, JHEP 1209 (2012) 035 [arXiv:1110.6926] [hep-ph]].

[11] R. Allahverdi, B. Dutta and K. Sinha, Phys. Rev. D 86 (2012) 095016 [arXiv:1208.0115 [hep-ph]].

[12] C. H. Chen, M. Drees and J. F. Gunion, Phys. Rev. Lett. 76 (1996) 2002 hep-ph/9512230, Addendum/erratum: hep-ph/9902309

[13] H. Baer, V. Barger and P. Huang, JHEP 1111 (2011) 031 arXiv:1107.5581 [hep-ph]].

[14] H. Baer, V. Barger, P. Huang and X. Tata, JHEP 1205 (2012) 109 arXiv:1203.5539 [hep-ph]].

[15] F. Brümmer and W. Buchmüller, JHEP 1107 (2011) 010 [arXiv:1105.0802 [hep-ph]].

[16] F. Brümmer and W. Buchmüller, JHEP 1205 (2012) 006 [arXiv:1201.4338 [hep-ph]].

[17] H. P. Nilles, S. Ramos-Sanchez, M. Ratz and P. K. S. Vaudrevange, Eur. Phys. J. C 59 (2009) 249 arXiv:0806.3905 [hep-th]].

[18] A. Maharana and E. Palti, Int. J. Mod. Phys. A 28 (2013) 1330005 arXiv:1212.0555 [hep-th]].

[19] H. E. Haber and G. L. Kane, Phys. Rept. 117 (1985) 75.

[20] A. Bartl, H. Fraas, W. Majerotto and N. Oshimo, Phys. Rev. D 40 (1989) 1594.; M. M. El Kheishen, A. A. Aboshousha and A. A. Shafik, Phys. Rev. D 45 (1992) 4345.; M. Guchait, Z. Phys. C 57 (1993) 157 [Erratum-ibid. C 61 (1994) 178]; G. J. Gounaris, C. Le Mouel and P. I. Porfyriadis, Phys. Rev. D 65 (2002) 035002 hep-ph/0107249].

[21] S. Y. Choi, J. Kalinowski, G. A. Moortgat-Pick and P. M. Zerwas, Eur. Phys. J. C 22 (2001) 563 [Addendum-ibid. C 23 (2002) 769] hep-ph/0108117.

[22] J. F. Gunion and H. E. Haber, Phys. Rev. D 37 (1988) 2515.

[23] S. Ambrosanio and B. Mele, Phys. Rev. D 55 (1997) 1399 [Erratum-ibid. D 56 (1997) 3157] hep-ph/9609212].

[24] S. Y. Choi, M. Guchait, J. Kalinowski and P. M. Zerwas, Phys. Lett. B 479 (2000) 235 hep-ph/0001175.

[25] A. Bharucha, A. Fowler, G. Moortgat-Pick and G. Weiglein, JHEP 1305 (2013) 053 arXiv:1211.3134 [hep-ph]].

[26] A. Bharucha, private communication.

[27] A. Bharucha, J. Kalinowski, G. Moortgat-Pick, K. Rolbiecki and G. Weiglein, Eur. Phys. J. C 73 (2013) 2446 arXiv:1211.3745 [hep-ph]].

[28] G. A. Moortgat-Pick and H. Fraas, Phys. Rev. D 59 (1999) 015016 hep-ph/9708481]; G. A. Moortgat-Pick, H. Fraas, A. Bartl and W. Majerotto, Eur. Phys. J. C 7 (1999) 113 hep-ph/9804306; G. A. Moortgat-Pick, H. Fraas, A. Bartl and W. Majerotto, Eur. Phys. J. C 9 (1999) 521 [Erratum-ibid. C 9 (1999) 549] hep-ph/9903220].

[29] H. E. Haber and D. Wyler, Nucl. Phys. B 323 (1989) 267.

[30] M. Berggren, T. Han, J. List, S. Padhi, S. Su and T. Tanabe, "Electroweakino Searches: A Comparative Study for LHC and ILC (A Snowmass White Paper)," arXiv:1309.7342 [hep-ph].

[31] K. Yokoya and P. Chen, Lect. Notes Phys. 400 (1992) 415.

[32] P. Chen, Phys. Rev. D 46 (1992) 1186.

[33] B. C. Allanach, Comput. Phys. Commun. 143 (2002) 305 hep-ph/0104145. 
[34] T. Fritzsche and W. Hollik, Eur. Phys. J. C 24 (2002) 619 hep-ph/0203159; T. Fritzsche, "Berechnung von Observablen zur supersymmetrischen Teilchenerzeugung an Hochenergie-Collidern unter Einschluss hoeherer Ordnungen", PhD Thesis, Cuvillier Verlag, Göttingen 2005, ISBN 3-86537-577-4.

[35] D. M. Pierce, J. A. Bagger, K. T. Matchev and R. -j. Zhang, Nucl. Phys. B 491 (1997) 3 hep-ph/9606211.

[36] W. Kilian, T. Ohl and J. Reuter, Eur. Phys. J. C 71 (2011) 1742 arXiv:0708.4233 [hep-ph]].

[37] T. Behnke, J. E. Brau, P. N. Burrows, J. Fuster, M. Peskin, M. Stanitzki, Y. Sugimoto and S. Yamada et al., "The International Linear Collider Technical Design Report - Volume 4: Detectors," (2013), pp. 32-36, arXiv:1306.6329 [physics.ins-det].

[38] M. Skrzypek and S. Jadach, Z. Phys. C 49 (1991) 577.

[39] C. Adolphsen, M. Barone, B. Barish, K. Büsser, P. Burrows, J. Carwardine, J. Clark and H. M. Durand et al., "The International Linear Collider Technical Design Report - Volume 3.II: Accelerator Baseline Design," (2013), pp. 8-10, arXiv:1306.6328 [physics.acc-ph].

[40] C. Hensel, "Search for nearly mass degenerate charginos and neutralinos in $e^{+} e^{-}$collisions," DESY-THESIS-2002-047. http://www-library.desy.de/cgi-bin/showprep.pl?desy-thesis-02-047

[41] T. Fritzsche and W. Hollik, Nucl. Phys. Proc. Suppl. 135 (2004) 102 hep-ph/0407095.

[42] W. Oller, H. Eberl and W. Majerotto, Phys. Rev. D 71 (2005) 115002 arXiv:hep-ph/0504109.

[43] W. Kilian, J. Reuter and T. Robens, Eur. Phys. J. C 48 (2006) 389 hep-ph/0607127.

[44] T. Robens, "Event Generation for Next to Leading Order Chargino Production at the International Linear Collider," hep-ph/0610401

[45] G. Moortgat-Pick, T. Abe, G. Alexander, B. Ananthanarayan, A. A. Babich, V. Bharadwaj, D. Barber and A. Bartl et al., Phys. Rept. 460 (2008) 131 hep-ph/0507011.

[46] K. Desch, J. Kalinowski, G. A. Moortgat-Pick, M. M. Nojiri and G. Polesello, JHEP 0402 (2004) 035 hep-ph/0312069.

[47] G. Moortgat-Pick, I. Fleck, S. Riemann, F. Simon, O. S. Adeyemi, G. Alexander, M. S. Amjad and V. V. Andreev et al., "Helmholtz Aliance Linear Collider Forum : Proceedings of the Workshops Hamburg, Munich, Hamburg 2010-2012, Germany," DESY 12-123H.

[48] D. Grellscheid and P. Richardson, arXiv:0710.1951 [hep-ph].

[49] M. Bahr, S. Gieseke, M. A. Gigg, D. Grellscheid, K. Hamilton, O. Latunde-Dada, S. Platzer and P. Richardson et al., Eur. Phys. J. C 58 (2008) 639 arXiv:0803.0883 [hep-ph]].

[50] T. Sjostrand, S. Mrenna and P. Z. Skands, JHEP 0605 (2006) 026 hep-ph/0603175].

[51] T. Behnke, J. E. Brau, P. N. Burrows, J. Fuster, M. Peskin, M. Stanitzki, Y. Sugimoto and S. Yamada et al., "The International Linear Collider Technical Design Report - Volume 4: Detectors," (2013), pp. 181-314, arXiv:1306.6329 [physics.ins-det].

[52] C. Bartels, M. Berggren and J. List, Eur. Phys. J. C 72 (2012) 2213 [arXiv:1206.6639 [hep-ex]].

[53] S. Agostinelli et al. [GEANT4 Collaboration], Nucl. Instrum. Meth. A 506 (2003) 250.

[54] T. Abe et al. [ILD Concept Group - Linear Collider Collaboration], "The International Large Detector: Letter of Intent," arXiv:1006.3396 [hep-ex].

[55] M. Berggren, "SGV 3.0 - a fast detector simulation," arXiv:1203.0217 [physics.ins-det].

[56] D. Schulte, "Study of Electromagnetic and Hadronic Background in the Interaction Region of the TESLA Collider," TESLA-97-08.

[57] P. Mora de Freitas and H. Videau, "Detector simulation with MOKKA / GEANT4: Present and future," LC-TOOL-2003010 .

[58] F. James and M. Roos, Comput. Phys. Commun. 10 (1975) 343. 